\title{
Stable immersions in orbifolds
}

\author{
ALDEN WALKER
}

\begin{abstract}
We prove that in any hyperbolic orbifold with one boundary component, the product of any hyperbolic fundamental group element with a sufficiently large multiple of the boundary is represented by a geodesic loop that virtually bounds an immersed surface. In the case that the orbifold is a disk, there are some conditions. Our results generalize work of Calegari-Louwsma and resolve a conjecture of Calegari.
\end{abstract}

20F65, 57M07; 57R42, 57R18

\section{Introduction}

It is an interesting and important problem to understand which curves on a surface bound an immersed subsurface. This paper addresses a question in this area primarily motivated by stable commutator length ( $\mathrm{scl}$ ) and quasimorphisms, and in this introduction, we provide some background. However, the main theorems are concerned only with immersions, so the reader can safely skim the portions of this introduction concerned with scl and retain a logically complete (though less colorful!) picture. For a more thorough scl background, especially as it relates to quasimorphisms and immersions, see Calegari [2] and Calegari and Louwsma [5].

\subsection{Orbifolds}

Recall that an orbifold is a space locally modeled on Euclidean space modulo finite groups of isometries. See Thurston [7] for background. In this paper, we will be concerned only with good orbifolds with a hyperbolic structure. By a hyperbolic orbifold $\Sigma$, we mean an orientable orbifold which arises as the quotient of hyperbolic space $\mathbb{H}^{2}$ by a finitely generated discrete subgroup $\Gamma \subseteq \operatorname{PSL}(2, \mathbb{R})$ such that $\Gamma$ acts properly on $\mathbb{H}^{2}$ and $\Gamma \backslash \mathbb{H}^{2}$ is finite-volume. Thus, $\mathbb{H}^{2}$ is the universal orbifold cover of $\Sigma$, and $\Gamma$ is identified with $\pi_{1}(\Sigma)$. We will use this notation throughout the paper.

Geometrically, a hyperbolic orbifold is a hyperbolic surface with finitely many cone points and cusps. We will be interested in how the hyperbolic structure can inform topological properties of $\Sigma$, so it is useful to also have a topological picture. Topologically, a hyperbolic orbifold is an orientable surface with finitely many points with a 
nontrivial structure (isotropy) group (which is always a finite cyclic group), and finitely many points removed. The underlying space of an orbifold $\Sigma$ is the topological space $\Gamma \backslash \mathbb{H}^{2}$ with the orbifold structure forgotten. A disk orbifold is a hyperbolic orbifold whose underlying space is a disk; that is, $\Sigma$ is topologically a sphere with cone points and a single removed point. There are various notations for orbifolds, which we will mostly avoid; however, following [5], we will refer to disk orbifolds with two cone points of orders $p$ and $q$ (and one cusp) as $(p, q, \infty)$ orbifolds. We clarify that this notation does not refer to a triangle group, which contains an orientation-reversing reflection. As an example, if we set $\Gamma=\operatorname{PSL}(2, \mathbb{Z})$, we get the modular orbifold, which is a $(2,3, \infty)$ orbifold.

While a hyperbolic orbifold technically has cusps instead of boundary, it still has natural boundary elements of the fundamental group, as follows. A small loop around a cusp gives a conjugacy class in the fundamental group $\pi_{1}(\Sigma)$. In the identification $\Gamma=\pi_{1}(\Sigma)$, this class is identified with the (parabolic) stabilizers of the preimages of the cusp in $\mathbb{H}^{2}$. Abusing notation, we will use $\partial \Sigma$ to mean either the union of the small loops around the cusps of $\Sigma$ or the union of the associated conjugacy classes in $\Gamma$, and we will refer to the loops as boundary components, or boundary loops, of $\Sigma$. As noted above, topologically, a hyperbolic orbifold is homotopy equivalent to an orbifold in which the cusps have been replaced with honest boundaries, motivating this nomenclature.

Remark 1.1 Just as in [5], the results and proofs in this paper apply equally well to hyperbolic orbifolds with geodesic boundaries instead of cusps, in which case the universal orbifold cover is not the entire hyperbolic plane. For simplicity, however, we will always use the definition of hyperbolic orbifold above.

\subsection{Immersions}

Let $S$ be a smooth surface, possibly with boundary and removed points, and let $\Sigma$ be a hyperbolic orbifold. If $f: S \rightarrow \Sigma$ is a continuous map, then we say that $f$ is an immersion if there is a lift to a map between universal covers $\widetilde{f}: \widetilde{S} \rightarrow \widetilde{\Sigma}=\mathbb{H}^{2}$ which is an immersion. Note that this is equivalent to saying that $f$ is an immersion away from the preimages of the cone points of $\Sigma$, and at the preimages of a cone point with angle $2 \pi / n, f$ has branch points of order exactly $n$. We will only be interested in orientation-preserving immersions, although the techniques in this paper apply to orientation-reversing ones as well.

If $\Sigma$ is a hyperbolic orbifold with fundamental group $\Gamma$, and $g \in \Gamma$ is a hyperbolic element, then $g$ is represented by a unique geodesic $\gamma \in \Sigma$. We say that $\gamma$ (or $g$ ) 
bounds an immersed surface if there is an oriented surface $S$ and an orientationpreserving immersion $f: S \rightarrow \Sigma$ such that $f(\partial S)=\gamma$ (as oriented 1-manifolds). We say that $\gamma$ (or $g$ ) virtually bounds an immersed surface if there is an oriented surface $S$ and an orientation-preserving immersion $f: S \rightarrow \Sigma$ such that $\left.f\right|_{\partial S}$ is a covering map $\partial S \rightarrow \gamma$. There are examples of curves on surfaces which do not bound an immersed surface but do virtually bound an immersed surface. We emphasize that a group element $g \in \Gamma$ only (virtually) bounds an immersed surface if the surface boundary maps to the geodesic representative of $g$.

\section{3 scl and stability}

One can make the analogous definition of virtually bounding an immersed surface for any homologically trivial 1-chain $C \in B_{1}(\Gamma)$, and in [2], Calegari shows the following stability theorem, simplified slightly here.

Theorem [2, Theorem C] Let $\Sigma$ be a compact, connected, orientable surface with boundary and $C \in B_{1}^{H}(\Gamma)$, where $\Gamma=\pi_{1}(\Sigma)$. Then for all sufficiently large $n$, the chain $n \partial \Sigma+C$ virtually bounds an immersed surface.

This theorem applies to orbifolds via lifting. Here

$$
B_{1}^{H}(\Gamma)=B_{1}(\Gamma) /\left\langle g^{n}=n g, h g h^{-1}=g\right\rangle
$$

is the space of homogenized 1-chains, which is more natural from the perspective of virtual immersions and scl. Stable commutator length is a norm on the vector space $B_{1}^{H}(\Gamma)$ (see [3] for background), and in [4], Calegari shows that the scl norm ball is polyhedral, in that its restriction to any finite-dimensional subspace is a rational finite-sided polyhedron. In [2], Calegari shows that there is a distinguished codimensionone so-called geometric face of the scl norm ball associated to the realization of the abstract group $\Gamma$ as the fundamental group of the orbifold $\Sigma$. This geometric face is dual to the rotation quasimorphism on $\Gamma$ induced by the circle action at infinity coming from the identification of $\Gamma=\pi_{1}(\Sigma) \subseteq \operatorname{PSL}(2, \mathbb{R})$. The 1 -chains projectively contained in the geometric face are exactly those which virtually bound immersed surfaces, so Theorem $\mathrm{C}$ is the main technical result showing that the geometric face is codimension-one.

So [2] provides a fundamental connection between (virtual) immersions, scl, and rotation quasimorphisms. Computer experiments led to the following conjecture:

Conjecture [2, Conjecture 3.16] Let $F=\langle a, b\rangle$ be a free group of rank 2. Let $w \in[F, F]$ be any homologically trivial word. Then for sufficiently large $n, w[a, b]^{n}$ virtually bounds an immersed surface in the realization of $F$ as the fundamental group of the hyperbolic once-punctured torus with boundary $[a, b]$. 
Note that [2, Conjecture 3.16] and [2, Theorem C] involve two similar, but definitely distinct, notions of stability; in Theorem $\mathrm{C}$, we are taking a formal sum with a multiple of the boundary, and in Conjecture 3.16, we are multiplying by it.

In [5], Calegari and Louwsma prove the analog of [2, Conjecture 3.16] for $(2, p, \infty)$ orbifolds:

Theorem [5, Theorem 3.1] Let $\Sigma$ be a $(2, p, \infty)$ orbifold with boundary loop $b$, and let $w \in \pi_{1}(\Sigma)$ be any hyperbolic element. Then for all sufficiently large $n, w b^{n}$ virtually bounds an immersed surface in $\Sigma$.

There is a potential ambiguity here, in that the boundary loop $b$ is not an element but a conjugacy class. But note that if the theorem holds for some representative of this conjugacy class, it holds for all of them, because the effect of changing representatives is essentially to change the word $w$. This is also true of the next theorem.

\subsection{Results}

In this paper, we generalize [5, Theorem 3.1] (see Remark 4.7, which addresses the issue of two vs three orbifold points) with the following theorem.

Theorem 4.1 Let $\Sigma$ be a hyperbolic orbifold whose underlying topological space is a disk and which has at least three orbifold points, of orders $\left\{o_{j}\right\}_{j=0}^{J-1}$. Let $w \in \Gamma=\pi_{1}(\Sigma)$ be any hyperbolic element, and let $b \in \Gamma$ be the boundary loop of $\Sigma$. Then there exists $N \in \mathbb{N}$ so that for all $n \geq 0$, the loop $w b^{N+n g}$ virtually bounds an immersed surface, where $g=\operatorname{gcd}\left(o_{0}-1, \ldots, o_{J-1}-1\right)$.

We also resolve [2, Conjecture 3.16], even in the presence of orbifold points:

Theorem 4.8 Let $\Sigma$ be a hyperbolic orbifold with one boundary component and with genus at least 1 . Let $\Gamma=\pi_{1}(\Sigma)$ with $b=\partial \Sigma \in \Gamma$, and let $w \in \Gamma$ be hyperbolic so that some power of $w$ is homologically trivial. Then there exists $N \in \mathbb{N}$ so that for all $n \geq 0$, the loop representing $w b^{N+n}$ virtually bounds an immersed surface.

In the course of proving these theorems, we also give a useful combinatorial certificate (Proposition 3.2) that a surface map into an orbifold $\Sigma$ is homotopic to an immersion with geodesic boundary, and hence a certificate that a collection of words in $\Gamma$ bounds an immersed surface. 


\subsection{Outline}

In Section 2, we review cyclic orders and realizations of a group as the fundamental group of a hyperbolic orbifold. In Section 3, we give a combinatorial parametrization of surface maps into orbifolds. In Section 4, we prove our main theorems.

Acknowledgments We wish to thank Danny Calegari, Joel Louwsma, Neil Hoffman, and especially the anonymous referee, who made suggestions which greatly improved the paper. Alden Walker was supported by NSF grant DMS 1203888.

\section{Hyperbolic orbifolds as realizations}

\subsection{Cyclic orders}

Informally, a cyclic order on a set $S$ is an arrangement of $S$ around a circle, and there are several equivalent ways of formalizing this. We define a cyclic order on a set $S$ to be a function $O: S \times S \times S \rightarrow\{-1,0,1\}$ which says whether a triple of elements is positively or negatively ordered (or 0 , if not all elements in the triple are distinct). A cyclic order must satisfy a compatibility condition on all 4-tuples of elements; namely if $O(x, y, z)=O(w, x, z)=1$, then $O(x, y, w)=O(y, z, w)=1$, the idea being that if we know $(x, y, z)$ and $(w, x, y)$ are positively ordered, then we can conclude that the four elements are arranged in the order $[x, y, z, w]$, and the cyclic order $O$ must respect this. As a shorthand for the function $O$, we will write cyclic orders in square brackets, as above, recording the (ordered) arrangement of the elements around a circle. A cyclic order given in square brackets is invariant under cyclic permutations of the list, and the function $O(x, y, z)$ can be computed by rotating the list so that $x$ is first; the value is then 1 if $y$ comes before $z$ and -1 otherwise.

If $T \subseteq S$ and $O_{T}$ and $O_{S}$ are cyclic orders on $T$ and $S$, respectively, then we say that $O_{T}$ and $O_{S}$ are compatible if $\left.O_{S}\right|_{T}=O_{T}$. If $T$ and $S$ are finite and the orders are written as cyclic lists with square brackets, then $O_{T}$ and $O_{S}$ are compatible if $O_{T}$ is obtained from $O_{S}$ by simply removing the elements of $S \backslash T$. For example, the orders $[a, c, b]$ and $[a, c, d, b]$ are compatible. See [1, Chapter 2].

\subsection{Realizations}

Let $\Sigma$ be a hyperbolic orbifold with one cusp, and let $\Gamma=\pi_{1}(\Sigma)$ be its fundamental group, so $\Gamma \subseteq \operatorname{PSL}(2, \mathbb{R})$ is a group of isometries. We now find a nice generating set for $\Gamma$. Let $R$ be a fundamental domain for the action, which is a polygon in $\mathbb{H}^{2}$ with some ideal vertices. The group $\Gamma$ is a free product of cyclic groups, which we write 
$\Gamma=\left(*_{i=0}^{I-1} Z_{i}\right) *\left(*_{j=0}^{J-1} C_{j}\right)$, where each $Z_{i}$ is infinite cyclic (ie a copy of $\left.\mathbb{Z}\right)$ and is generated by $z_{i}$, and each $C_{j}$ is finite cyclic and is generated by $c_{j}$ with order $o_{j}$. The $z_{i}$ are hyperbolic, and the $c_{j}$ are elliptic. After conjugation, we may assume that the axes of the $z_{i}$ all pass through $R$, and the fixed points of the $c_{j}$ are all vertices of $R$. It is possible that there is more than one choice for the $c_{j}$, since a single orbifold point may appear multiple times as a vertex of $R$. Any of the options will work. We may also assume that all the $c_{j}$ rotate counterclockwise. We will always write words in $\Gamma$ using positive powers of the $c_{j}$. For a given word $w$ in the given generators of $\Gamma$, we will call a specific generator at a specific location in $w$ a letter, and we'll denote the letter in $w$ at position $k$ by $w_{k}$, with indices starting at 0 .

We call the orbifold $\Sigma$ together with the generating set $\left\{c_{0}, \ldots, c_{J-1}, z_{0}, \ldots, z_{I-1}\right\}$ chosen as above a realization of the abstract group $\left(*_{i=0}^{I-1} Z_{i}\right) *\left(*_{j=0}^{J-1} C_{j}\right)$, and we will always assume that our hyperbolic orbifolds come with such a generating set.

For each $z_{i}$, mark the intersections of the hyperbolic axis of $z_{i}$ with the boundary of $R$ : the initial intersection with $z_{i}^{-1}$ and the terminal intersection with $z_{i}$. Also mark the elliptic fixed point of $c_{j}$ by $c_{j}$. Reading the boundary of $R$ counterclockwise, this induces a cyclic order on the set of generators

$$
S=\left\{z_{0}, z_{0}^{-1}, \ldots, z_{I-1}, z_{I-1}^{-1}, c_{0}, \ldots, c_{J-1}\right\} .
$$

Note that the set $S$ contains each $z_{i}$ and its inverse, but only the positive power of $c_{j}$. We'll denote the cyclic order on $S$ by $O_{\Sigma}$.

It may seem as though there is potential ambiguity in the cyclic order $O_{\Sigma}$, because some elliptic $c_{j}$ may have been associated with multiple vertices of $R$, and we chose the vertex to be labeled arbitrarily. However, note that if we were to choose a different vertex, that would correspond to choosing a different (conjugate) generator in $\Gamma$, so while we would get a different cyclic order, it's also a genuinely different identification with $\left(*_{i=0}^{I-1} Z_{i}\right) *\left(*_{j=0}^{J-1} C_{j}\right)$.

Example 2.1 Figure 1 shows the fundamental domain $R$ for a surface of genus one with one boundary component and three orbifold points. Here all the orders $o_{j}$ are 4 . To illustrate how the generators act, we've numbered the images of the point marked 0 under the successive subwords of the boundary word $c_{0} z_{0}^{-1} z_{1}^{-1} c_{1} c_{2} z_{0} z_{1}$. Recall $\Gamma$ acts on $\mathbb{H}^{2}$ on the left, so the successive subwords are suffixes of the boundary word. The induced cyclic order $O_{\Sigma}$ can be read off counterclockwise from the boundary of $R$, and it is $\left[c_{1}, c_{2}, z_{0}, z_{1}^{-1}, c_{0}, z_{0}^{-1}, z_{1}\right]$. Figure 2 shows what the orbifold looks like, topologically. Note it is easy to read off the boundary word and the cyclic order from Figure 2. 


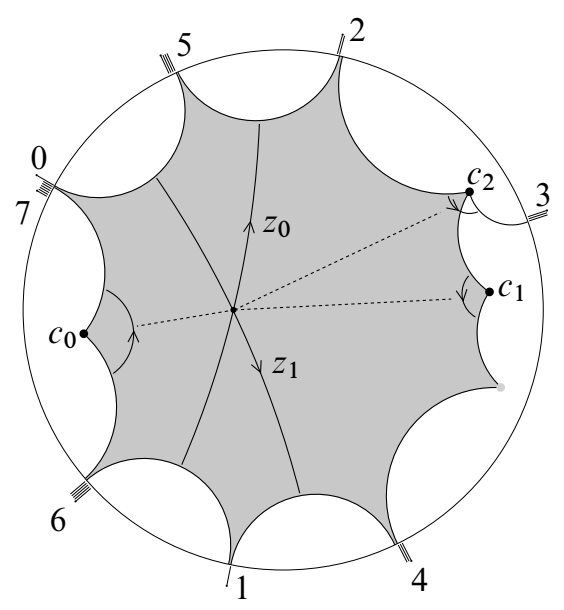

Figure 1: A fundamental domain for an orbifold, as described in Example 2.1

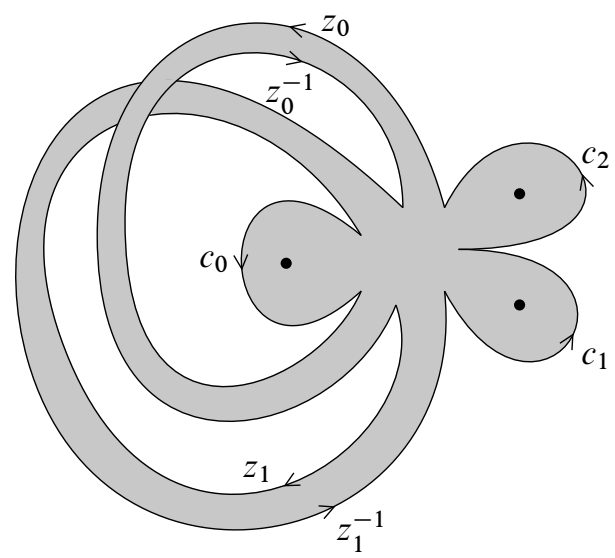

Figure 2: The orbifold from Figure 1, topologically, with the loops representing the generators of $\Gamma$. Note the boundary word is easy to read off. The cyclic order $O_{\Sigma}$ on generators is the cyclic order around the central part of the surface. See Example 2.1.

\subsection{Core graphs of realizations}

Let $\Sigma$ be a realization of $\Gamma$ with generating set $\left\{c_{0}, \ldots, c_{J-1}, z_{0}, \ldots, z_{I-1}\right\}$ and fundamental domain $R$, as above. We will use the choice of generators and fundamental domain to define a graph on the orbifold $\Sigma$, as follows. Recall that as part of the realization, we have points on the boundary of $R$ labeled by the elements of $S=$ $\left\{z_{0}, z_{0}^{-1}, \ldots, z_{I-1}, z_{I-1}^{-1}, c_{0}, \ldots, c_{J-1}\right\}$. These points induce the cyclic order $O_{\Sigma}$ on $S$. 
Let $p$ be a point in the interior of $R$ (a more central point makes a nicer picture, but it doesn't matter where it is). Construct a directed graph $G_{\Sigma}^{\prime}$ on $R$ with vertex set $\{p\} \cup S$ with edges as follows: for each vertex $c_{j}$ in $S$, there is an edge from $p$ to $c_{j}$. For each vertex $z_{i}$, there is an edge from $p$ to $z_{i}$, and for each vertex $z_{i}^{-1}$, there is an edge from $z_{i}^{-1}$ to $p$. These edges can be made all embedded and disjoint in $R: R$ is topologically a disk, so we can clearly connect an interior point to arbitrary points on the boundary with a series of disjoint, embedded arcs. For example, we could make them geodesic arcs.

Now let $G_{\Sigma}$ be the quotient of $G_{\Sigma}^{\prime}$, which is a graph in $\Sigma$. The graph $G_{\Sigma}$ is the core graph of the orbifold $\Sigma$ realizing $\Gamma$. We now describe $G_{\Sigma}$ and name its parts so we can refer to them later. The quotient map from $R$ to $\Sigma$ is an embedding away from the boundary, so to know what $G_{\Sigma}$ is, it suffices to consider what happens to the vertices $z_{i}^{ \pm 1}$ and $c_{j}$. The $c_{j}$ vertices in $G_{\Sigma}^{\prime}$ are each sent to one of the cone points. The pair of vertices $z_{i}^{ \pm 1}$ in $G_{\Sigma}^{\prime}$ are identified into a single vertex in $G_{\Sigma}$, which we will denote by $z_{i}$. So the vertex set of the core graph $G_{\Sigma}$ is $\left\{p, z_{0}, \ldots, z_{I-1}, c_{0}, \ldots, c_{J-1}\right\}$. For each $i$, there is an edge from $p$ to $z_{i}$ and from $z_{i}$ to $p$, and for each $j$, there is an edge from $p$ to $c_{j}$. Figure 3 shows the core graph for the orbifold given in Example 2.1.
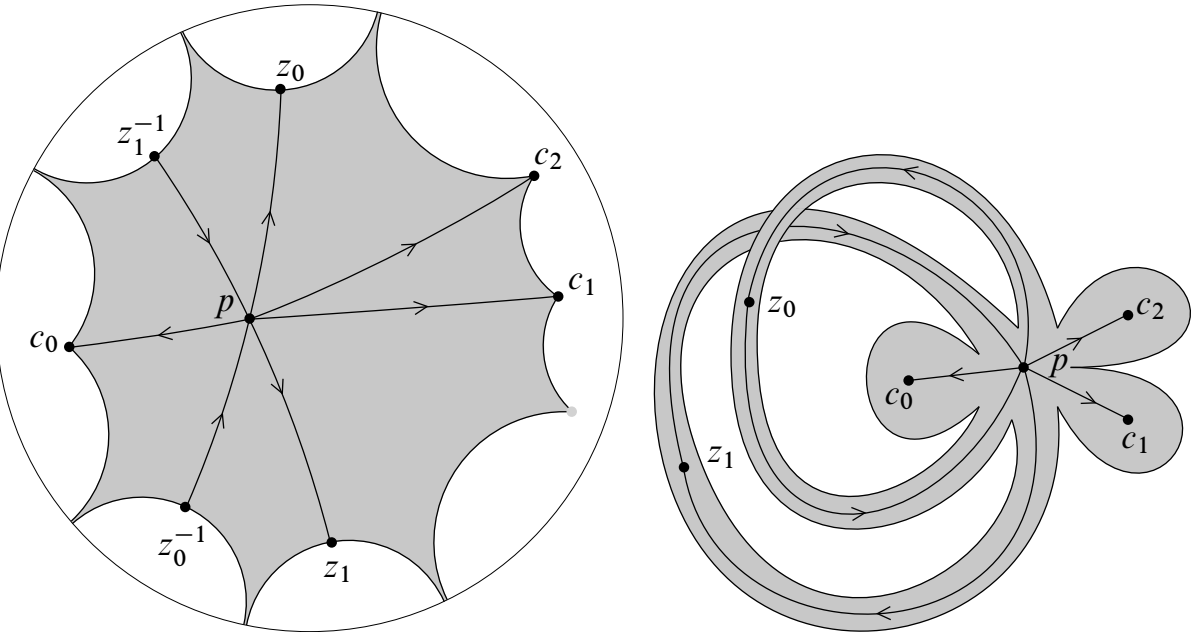

Figure 3: The core graph of the realization described in Example 2.1. Left, the graph $G_{\Sigma}^{\prime}$ is shown drawn in the fundamental domain $R$. Right, the core graph $G_{\Sigma}$ is shown on the orbifold, drawn as in Figure 2.

Consider the vertex $p$ in the graph $G_{\Sigma}$. Since the graph $G_{\Sigma}$ comes with an embedding in $\Sigma$, the vertex $p$ has a cyclic order on the incident edges obtained by simply reading the directed labels in counterclockwise order around $p$ (where the incoming edge from 
$z_{i}$ to $p$ is read as $z_{i}^{-1}$ ). Note that these labels are exactly $S$, and this cyclic order is exactly $O_{\Sigma}$.

\subsection{Covering trees of core graphs}

The core graph of the hyperbolic orbifold $\Sigma$ realizing $\Gamma$ is a graph $G_{\Sigma}$ embedded in $\Sigma$. The preimage $\widetilde{G}_{\Sigma}$ of $G_{\Sigma}$ in the universal cover $\widetilde{\Sigma}=\mathbb{H}^{2}$ is a graph in $\mathbb{H}^{2}$. We call this graph the covering tree of $G_{\Sigma}$. We will verify momentarily that it is, in fact, a tree. In $G_{\Sigma}$, each vertex $c_{j}$ has just a single incoming edge. In the covering tree, the preimages of the vertex $c_{j}$ have $o_{j}$ incoming edges, where recall $o_{j}$ is the order of the generator $c_{j}$. This is quite natural, since the covering map $\mathbb{H}^{2} \rightarrow \Sigma$ branches at the preimages of the cone points. See Figure 4.

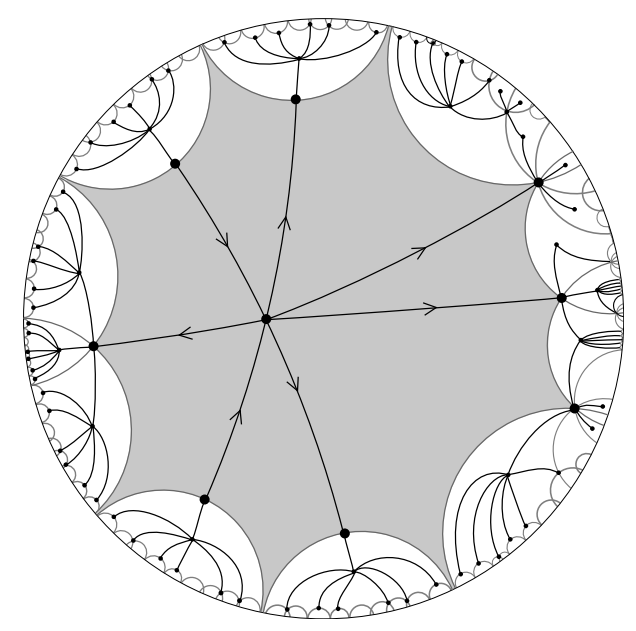

Figure 4: The covering tree of the core graph in Figure 3

Since $G_{\Sigma}$ is embedded in $\Sigma$, the preimage $\widetilde{G}_{\Sigma}$ is embedded in $\mathbb{H}^{2}$. The fact that $\widetilde{G}_{\Sigma}$ is a tree is quite straightforward to see intuitively, since $\mathbb{H}^{2}$ is the universal cover of $\Sigma$, and $G_{\Sigma}$ carries part of the fundamental group, but we go through it carefully. To see that it is a tree, suppose that we have a loop $\gamma$ in $\widetilde{G}_{\Sigma}$. Now, $\gamma$ must pass through a preimage of the vertex $p$ since the vertices $z_{i}$ and $c_{j}$ are connected only to $p$ in $G_{\Sigma}$. Reading the vertex labels around $\gamma$ gives a word in $\Gamma$ taking a preimage of $p$ to itself (where each time we pass through a preimage of $c_{j}$, we must choose the appropriate power of $c_{j}$ to obtain the desired angle, and each time we pass through $z_{i}$, we record $z_{i}$ or $z_{i}^{-1}$, depending on whether we crossed the edges adjacent to $z_{i}$ respecting the direction). Any word in $\Gamma$ taking a preimage of $p$ to itself is trivial, so it must be (a conjugate of) the word $c_{j}^{n o_{j}}$ for some $n$. But this word produces a trivial path, so $\gamma$ is a trivial loop, and we see that $\widetilde{G}_{\Sigma}$ must be a tree. 


\section{Cyclic fatgraphs and immersions in orbifolds}

\subsection{Cyclic fatgraphs}

Our proofs will build surface immersions using cyclic fatgraphs over $\Gamma$, which are combinatorialized surface maps into $\Sigma$. A cyclic fatgraph over $\Gamma$ is a surface which is built out of pieces, which are rectangles, polygons, and group polygons, and where the pieces are glued along edges. We now define all these terms.

A rectangle is a 2-cell whose boundary is an oriented simplicial loop with four 1simplices. We think of a rectangle as a rectangular strip. It is labeled on one long side by an infinite order generator $z_{i}$ and on the other by its inverse $z_{i}^{-1}$. The notation for such a rectangle is $r\left(z_{i}\right)$. The short 1-simplices of the rectangle $r\left(z_{i}\right)$ are rectangle edges, and a rectangle edge is denoted by $\operatorname{re}\left(z_{i}\right)$ or $\operatorname{re}\left(z_{i}^{-1}\right)$ depending on which long labeled side comes after the rectangle edge. See Figure 5.

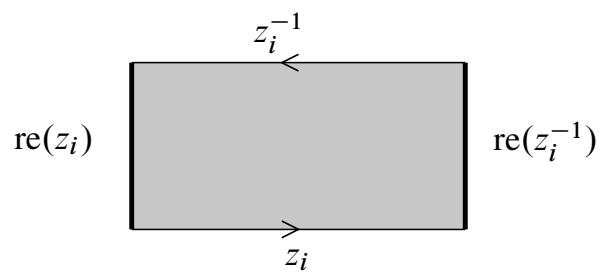

Figure 5: A rectangle, with two labeled sides and two edges (bold)

A group polygon is a 2-cell whose boundary is an oriented simplicial loop with simplices alternating between labeled sides and group polygon edges. The labeled sides are all labeled by the same finite order generator, and there must be exactly as many labeled sides as the order $o_{j}$ of $c_{j}$. Every group polygon edge in such a group polygon is denoted by $\operatorname{ge}\left(c_{j}\right)$. The notation for this group polygon is $g\left(c_{j}\right)$. See Figure 6 .

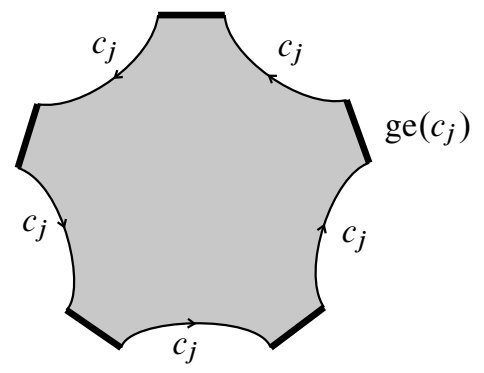

Figure 6: A group polygon in the case that $o_{j}=5$

A polygon is a 2-cell whose boundary is an oriented simplicial complex whose simplices are all polygon edges. A polygon edge can be one of $\operatorname{pe}\left(c_{j}\right), \operatorname{pe}\left(z_{i}\right)$, or $\operatorname{pe}\left(z_{i}^{-1}\right)$. There 
is a restriction that a polygon must be locally reduced, which means that pe $\left(z_{i}\right)$ cannot immediately follow pe $\left(z_{i}\right)$, and similarly for the inverses. A nondegenerate polygon can have two or more edges. We will often refer to polygons with the name appropriate to their number of edges, for example bigon, triangle, square, etc. See Figure 7.

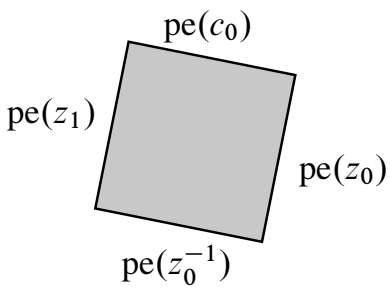

Figure 7: A polygon. All the sides are edges. This square appears in the cyclic fatgraph shown in Figure 10.

For technical reasons, it is convenient to allow polygons with a single edge (a monogon). Such polygons may only have edges of the form $\mathrm{pe}\left(c_{j}\right)$ (a finite-order generator). Monogons are needed to allow repeated copies of $c_{j}$ to appear on the boundary of a cyclic fatgraph. Figure 10 contains an example.

When dealing with these pieces, we will often refer to finite-order or infinite-order edges, meaning rectangle, group polygon, and polygon edges, as appropriate.

A cyclic fatgraph over $\Gamma$ is a surface with a simplicial structure such that every 2-cell has the structure of a rectangle, polygon, or group polygon. If a 1-simplex is the boundary of two 2-cells, then one of the 2-cells must be a polygon and the other must be a rectangle or group polygon, and the simplex of intersection must be an edge in both, and the labels must match, eg pe $\left(z_{i}\right)$ is glued to re $\left(z_{i}\right)$. That is, a cyclic fatgraph over $\Gamma$ is a surface built out of rectangles and group polygons by gluing them together around polygons along edges. See Figure 8.

In our drawings, including Figure 8, note that where the rectangles and group polygons appear to attach directly to each other, there is technically a bigon (polygon with two sides) joining them. This technicality is useful to avoid special cases in the definition and for some definitions to follow.

\subsection{Spines of cyclic fatgraphs}

Given a cyclic fatgraph $Y$, we define the spine $G_{Y}$ of $Y$, which is a directed graph on $Y$, to be the graph dual to the cellulation of $Y$ by the polygons, rectangles, and group polygons in $Y$. Since there is a vertex for each piece, we call the vertices polygon, rectangle, or group polygon vertices as appropriate. Orient the edges of $G_{Y}$ so that 


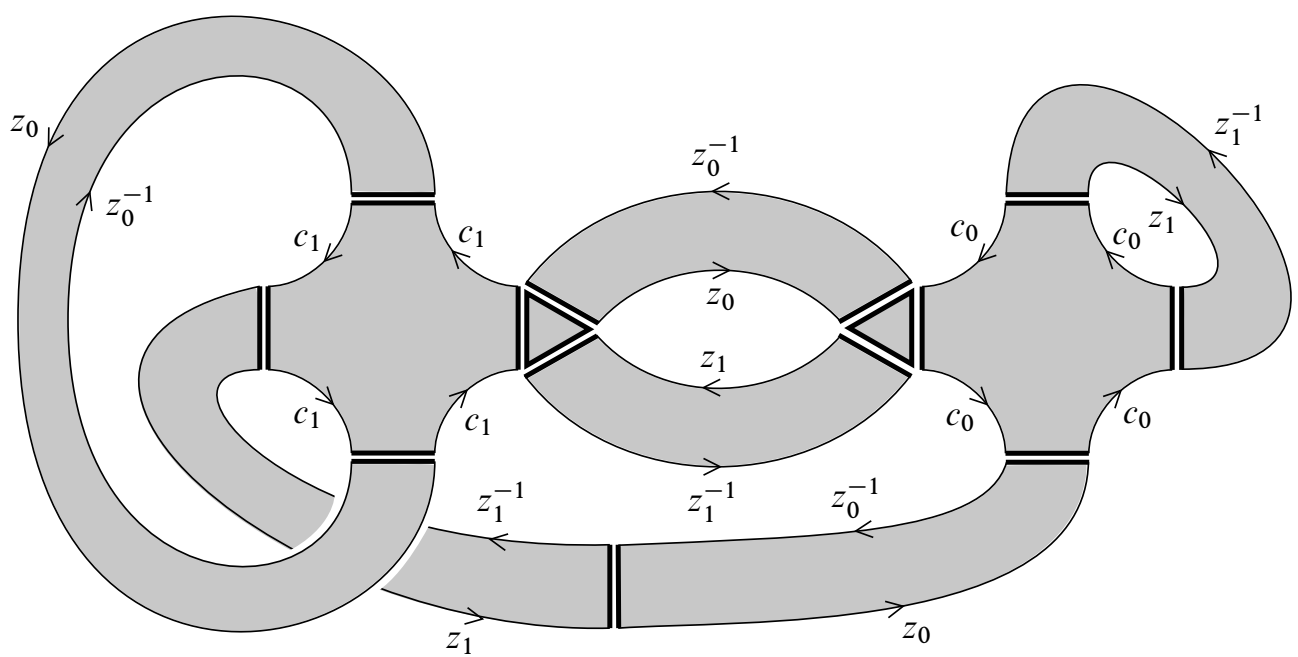

Figure 8: A cyclic fatgraph over the group $\Gamma$ from Example 2.1. Recall that $o_{j}=4$ for all $j$, so each group polygon has four sides. This fatgraph has boundary $z_{0} c_{0} z_{1}^{-1} c_{0} z_{0}^{-1} c_{1} z_{0} c_{1} z_{1}^{-1} c_{0} z_{0}^{-1} z_{1}^{-1} c_{1} z_{0}^{-1} c_{1} z_{1}+z_{0} z_{1}+z_{1} c_{0}$. There are seven bigons and two triangles.

every edge between a polygon vertex and a group polygon vertex is directed away from the polygon vertex. Orient the two edges incident to a rectangle vertex so that their orientation agrees with the orientation on the side of the rectangle labeled by $z_{i}$ (and against the orientation on the side labeled by $z_{i}^{-1}$ ). Note that $Y$ deformation retracts to its spine. See Figure 9.

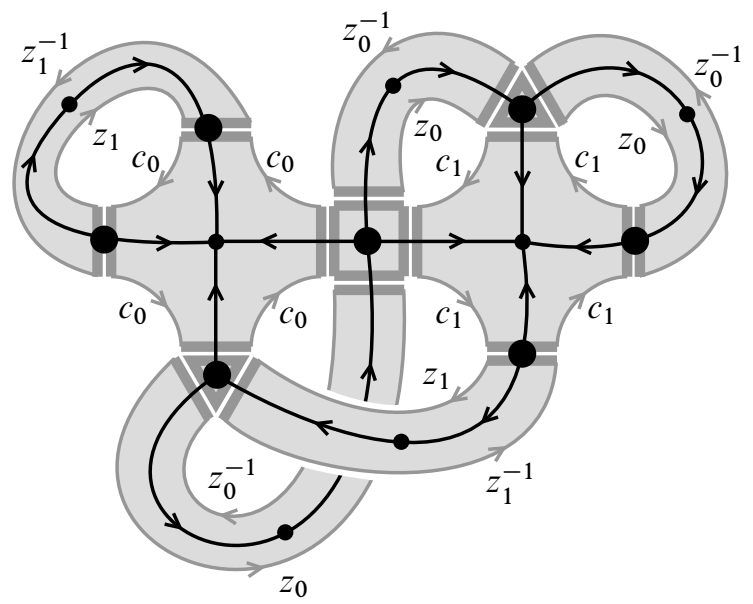

Figure 9: The spine of a cyclic fatgraph. The polygon vertices are drawn larger to differentiate them from the rectangle and group polygons vertices. Note there are four polygon vertices in the (invisible) bigons. 


\subsection{Immersed surfaces in orbifolds}

Let $\Sigma$ be an orbifold realizing the group $\Gamma$ with core graph $G_{\Sigma}$, and suppose that we have a cyclic fatgraph $Y$ over $\Gamma$, as defined above. There is a natural simplicial map from the spine $G_{Y}$ of $Y$ to the core graph $G_{\Sigma}$ defined by sending the polygon, rectangle, and group polygon vertices of $G_{Y}$ to the $p, z_{i}$, and $c_{j}$ vertices of $G_{\Sigma}$, and by mapping the edges so as to preserve orientation. Let $f_{Y}: Y \rightarrow \Sigma$ be the map deformation retracting $Y$ to its spine, and then mapping $G_{Y}$ to $G_{\Sigma}$ as above. We call this map the fatgraph map induced by $Y$. Note that $f_{Y}$ is a map of a surface with boundary into the orbifold $\Sigma$. Though we will not need it, we remark that every map of a surface factors through a fatgraph map:

Lemma 3.1 [8, Lemma 4.4] After compression and homotopy, every surface map $f: S \rightarrow \Sigma$ factors as $f_{Y} \circ i$, where $i: S \rightarrow Y$ is a homeomorphism between $S$ and a cyclic fatgraph over $\Gamma$, and $f_{Y}$ is the fatgraph map.

In [8], the lemma is not stated exactly in this way, but it follows from the proof. In the case of free groups, this lemma is due to Culler [6].

The main result of this section is that there is a local combinatorial certificate that the map $f_{Y}$ is homotopic to an immersion with geodesic boundary. We now describe this certificate.

Recall that $S$ is the set of generators $\left\{z_{0}, z_{0}^{-1}, \ldots, z_{I-1}, z_{I-1}^{-1}, c_{0}, \ldots, c_{J-1}\right\}$ of $\Gamma$, and $O_{\Sigma}$ is the cyclic order on $S$ determined by the realization $\Sigma$. Let us be given a polygon $P$ in a cyclic fatgraph over $\Gamma$. The edges of $P$ have an intrinsic cyclic order, so the set of labels on the edges of $P$ is a cyclically ordered multiset. The cyclically ordered multiset of labels of the edges of $P$ will be denoted by $\partial P$. For example, if the edges of $P$ are $\left[\operatorname{pe}\left(z_{0}^{-1}\right), \operatorname{pe}\left(c_{0}\right), \operatorname{pe}\left(c_{1}\right), \operatorname{pe}\left(z_{1}^{-1}\right), \operatorname{pe}\left(c_{0}\right)\right]$, then the set of labels is the cyclically ordered multiset $\partial P=\left[z_{0}^{-1}, c_{0}, c_{1}, z_{1}^{-1}, c_{0}\right]$. We call a polygon small if $\partial S$ is actually a set; ie if each label appears at most once in $\partial P$. Notice that if $P$ is small, then $\partial P \subseteq S$, so there are two cyclic orders on $\partial P$ : its intrinsic cyclic order and the cyclic order given by $O_{\Sigma}$.

There is a special polygon, the standard $\Sigma$ polygon, which is the polygon such that $\partial P=O_{\Sigma}$, ie every outgoing edge appears exactly once, and in the cyclic order $O_{\Sigma}$. The standard $\Sigma$ polygon is the largest polygon whose boundary is compatibly ordered with $O_{\Sigma}$.

Proposition 3.2 Let $Y$ be a cyclic fatgraph over $\Gamma$ with induced surface map $f_{Y}: Y \rightarrow$ $\Sigma$. Suppose that every boundary component of $Y$ is realized in $\Sigma$ by a geodesic loop 
(is not finite order or parabolic). If every polygon $P$ in $Y$ has the property that $P$ is small and the cyclic order on $\partial P$ is compatible with $O_{\Sigma}$, then $f_{Y}$ is homotopic to an immersion with geodesic boundary.

Remark 3.3 Cyclic orders are useful for many geometric things; see [1, Chapter 2] and [3, Section 4.2.5]. Proposition 3.2 is essentially a generalization of the ideas in [3, Section 4.2.5] to orbifolds.

Remark 3.4 Consider Figure 9, the spine of a fatgraph and Figure 4, the covering tree of the core graph of a realization. The map $f_{Y}$ retracts the fatgraph to the spine and then sends the spine inside the core graph. In the universal cover, then, the covering map $\widetilde{f_{Y}}$ sends the universal cover of the spine inside the covering tree of the core graph. The hypothesis of Proposition 3.2 makes sure that this covering map preserves the cyclic orders of the edges around each vertex and is thus an embedding. Since the universal cover of the fatgraph retracts to the spine and the spine is embedded, Proposition 3.2 is quite natural. The proof is a formalization of this.

Proof of Proposition 3.2 In order to prove that $f_{Y}$ is homotopic to an immersion, we must show that the lift $\widetilde{f_{Y}}: \widetilde{Y} \rightarrow \widetilde{\Sigma}$ is homotopic to an immersion, where the homotopy of $\widetilde{f_{Y}}$ must be equivariant with respect to $\pi_{1}(Y)$.

Write $\widetilde{f_{Y}}=g \circ h$, where $h$ is the deformation $\widetilde{Y} \rightarrow \widetilde{G_{Y}}$, and $g$ is the simplicial graph map $\widetilde{G_{Y}} \rightarrow \widetilde{G_{\Sigma}}$. Let us consider what happens to the stars of the vertices under the graph map $g$. There are three kinds of vertices in $\widetilde{G_{Y}}$, covering rectangle, group polygon, and polygon vertices. The stars of rectangle vertices are 2-valent and map to the 2 -valent stars of the $z_{i}$ vertices in $\widetilde{G_{\Sigma}}$. A group polygon vertex $v$ corresponding to $c_{j}$ has valence $o_{j}$ and maps to a vertex $w$ covering a torsion vertex $c_{j}$ of the core graph. The vertices $v$ and $w$ each have $o_{j}$ incoming edges, and because the star of $v$ covers the 1 -valent star of the projection of $w$ with degree $o_{j}$, the $o_{j}$-valent star of $v$ is identified with the $o_{j}-$ valent star of $w$. This uses the fact that the graphs are embedded in the surfaces $Y$ and $\Sigma$; there is no angle structure on an abstract graph, but there is for the graphs $G_{Y}$ and $G_{\Sigma}$, so we know how the cone points are covered.

Finally, consider the star of a polygon vertex $v$ in $\widetilde{G_{Y}}$, which maps to a vertex $w$ covering $p$ in $G_{\Sigma}$. By assumption, the multiset of incident edge labels at $v$ is a subset of the incident edges at $w$ (the polygon is small), and they are compatibly cyclically ordered (the cyclic order on the polygon is compatible with $O_{\Sigma}$ ). Therefore, the star of $v$ is embedded in the star of $w$.

We conclude that the map $g$ embeds the tree $\widetilde{G_{Y}}$ inside the tree $\widetilde{G_{\Sigma}}$ in a way that preserves the cyclic order on every vertex. If the reader is familiar with pleated surfaces 
(see [7]), it is enough now to note that this fact about graphs implies that the pleated surface representative of the map $f_{Y}$ has only positive simplices and is therefore an immersion. If not, we explain. Give $Y$ a hyperbolic structure with geodesic boundary and decompose it into ideal triangles (which will necessarily be spun around some closed geodesics in $Y$ ). These ideal triangles lift to ideal triangles in the universal cover $\widetilde{Y}$. Because $h$ is a deformation retraction to a tree, the image of an ideal triangle $T$ under $h$ is an infinite tripod $h(T)$, and because $g$ embeds the tree $\widetilde{G_{Y}}$ inside $\widetilde{G_{\Sigma}}$, the image of $T$ under $g \circ h=\widetilde{f_{Y}}$ is an infinite tripod $g(h(T))$. Now, $g$ sends the three ideal points at the ends of $h(T)$ to the three ideal points at the ends of $g(h(T))$. And because $g$ preserves the cyclic order on every vertex, the cyclic orders on these triples of points are the same.

Therefore, the image of $T$ under $\widetilde{f_{Y}}$ is an infinite tripod whose ends have the same cyclic order as the ends of $T$. There is a geodesic ideal triangle $T^{\prime}$ in $\widetilde{\Sigma}$ with the same ends as $\widetilde{f_{Y}}(T)$, and we can homotope $\widetilde{f_{Y}}$ on $T$ to map $T$ to $T^{\prime}$. Because the order on the ends is preserved, this map is orientation-preserving.

Do this homotopy on lifts of each ideal triangle in $Y$, and extend the homotopy equivariantly over $\widetilde{Y}$. The result is an equivariant homotopy of $\widetilde{f_{Y}}$ to a map which takes ideal triangles to ideal triangles in an orientation-preserving way; that is, it is an immersion taking geodesic boundary to geodesic boundary.

Example 3.5 While Proposition 3.2 may seem technical, it is straightforward to apply in practice. Consider the orbifold $\Sigma$ from Example 2.1 with fundamental group $\Gamma$. Figure 9 shows a cyclic fatgraph $Y$ over $\Gamma$. A simple check at the polygons shows that the cyclic orders are $\left[c_{1}, z_{0}, c_{0}, z_{0}^{-1}\right],\left[c_{1}, z_{0}, z_{0}^{-1}\right],\left[c_{0}, z_{0}, z_{1}^{-1}\right],\left[c_{1}, z_{0}^{-1}\right],\left[c_{1}, z_{1}\right]$, $\left[c_{0}, z_{1}^{-1}\right]$, and $\left[c_{0}, z_{1}\right]$, which are all compatible with $O_{\Sigma}$. Thus, the map $f_{Y}$ can be straightened to an immersion with geodesic boundary, and in particular, the boundary loops bound an immersed surface.

\subsection{Building immersed surfaces}

Given a fatgraph, it is easy to check using Proposition 3.2 whether the induced surface map is homotopic to an immersion with geodesic boundary. Our goal in this paper is to build fatgraphs (1) which are homotopic to immersions with geodesic boundary and (2) which have some given word in the generators as a boundary. In this section, we show that relaxing either of these conditions makes the problem trivial. This section is mainly background and introduction to the methods we will use later. 
3.4.1 Cyclic fatgraphs which satisfy Proposition 3.2 Constructing fatgraphs which satisfy the hypotheses of Proposition 3.2 is quite straightforward. The proposition requires that the fatgraph be built using only small polygons whose intrinsic cyclic order on edges is compatible with $O_{\Sigma}$. So if we simply enumerate all possible polygons satisfying this hypothesis, and all rectangles and group polygons, then we can take any subset of these pieces such that each edge occurs the same number of times in polygons as it does in rectangles and group polygons and then glue these pieces together arbitrarily.

3.4.2 Cyclic fatgraphs over $\boldsymbol{\Gamma}$ with given boundary $\boldsymbol{w}$ The boundary $\gamma$ of $Y$ has a simplicial structure, and every (oriented) 1-simplex in $\gamma$ is labeled by a generator inherited from the labels on the pieces of $Y$. If we simply read off the labels as we follow $\gamma$, that tells us the image word $f_{Y}(\gamma)$ in $\Gamma$.
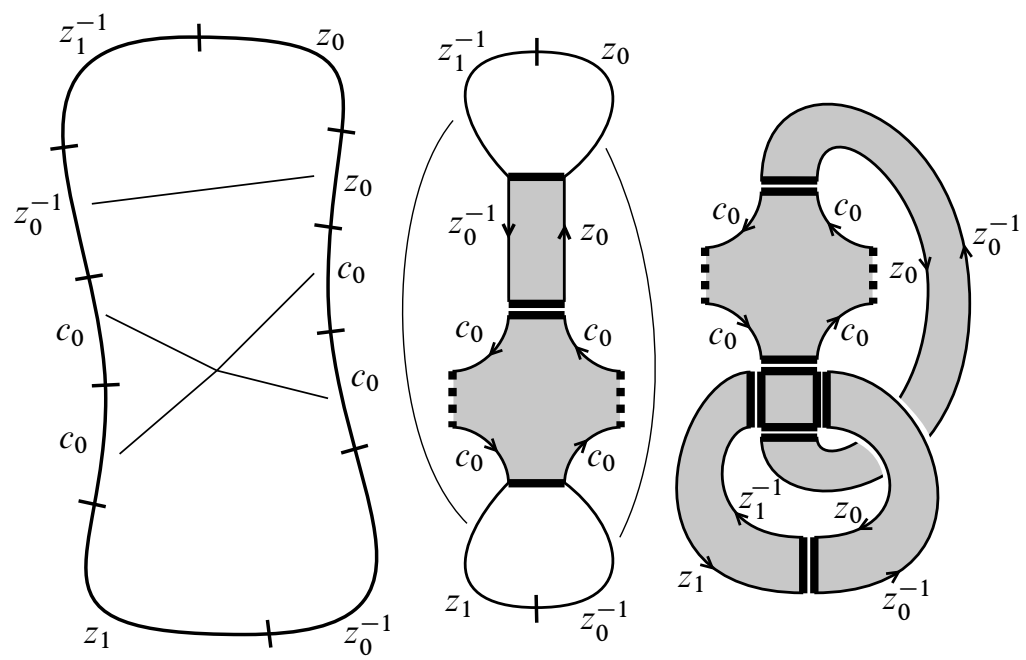

Figure 10: To pinch the loop $c_{0}^{2} z_{0}^{2} z_{1}^{-1} z_{0}^{-1} c_{0}^{2} z_{1} z_{0}^{-1}$ into a cyclic fatgraph, we can pair up the letters arbitrarily into rectangles and group polygons. The group polygon has two group polygon edges which are glued to monogons, which allows $c_{0}^{2}$ to be part of the boundary.

To build a surface map into $\Sigma$ with a desired boundary loop $w$, which must of course be homologically trivial, we can start with an oriented simplicial circle, with each simplex labeled by a generator, such that the circle reads off $w$. Because $w$ is homologically trivial, there are as many instances of $z_{i}$ as there are of $z_{i}^{-1}$, so we can pair them arbitrarily into rectangles. Similarly, the $c_{j}$ must come in groups of $o_{j}$, so we can group them together to form group polygons. Placing polygons at the junctions of the group polygons and rectangles to fill in the holes, we produce a cyclic fatgraph $Y$ over 
$\Gamma$, which comes with the surface map $f_{Y}: Y \rightarrow \Sigma$. By construction, the boundary of $Y$ maps to $w$. This construction yields a surface map bounding any homologically trivial word, or words, in $\Gamma$. See Figure 10.

3.4.3 Summary We have seen that building a fatgraph satisfying Proposition 3.2 is easy, and building a fatgraph with a given boundary is easy. However, the methods to accomplish each goal are very different, and note that if we glue pieces as in Section 3.4.1, it is quite difficult to control what the boundary is, and if we pinch a boundary loop as in Section 3.4.2, it is quite difficult to control what polygons appear.

The proofs of Theorems 4.1 and 4.8 use Section 3.4.1, but done carefully in a way which controls the boundary.

\section{Stability}

\subsection{Disk orbifolds}

In this section, we prove our main result, which says that, under some conditions, the product of any word in $\Gamma$ with a sufficiently high multiple of the boundary word of $\Sigma$ is a loop which bounds an immersed surface with geodesic boundary in $\Sigma$. First, we state and prove the version for orbifolds whose underlying topological space is a disk.

Theorem 4.1 Let $\Sigma$ be a hyperbolic orbifold whose underlying topological space is a disk and which has at least three orbifold points, of orders $\left\{o_{j}\right\}_{j=0}^{J-1}$. Let $w \in \Gamma=\pi_{1}(\Sigma)$ be any hyperbolic element, and let $b \in \Gamma$ be the boundary loop of $\Sigma$. Then there exists $N \in \mathbb{N}$ so that for all $n \geq 0$, the loop $w b^{N+n g}$ virtually bounds an immersed surface, where $g=\operatorname{gcd}\left(o_{0}-1, \ldots, o_{J-1}-1\right)$.

Remark 4.2 As mentioned in the introduction, the boundary of the orbifold is actually associated to a conjugacy class in $\Gamma$, not a specific word, so taking $w b^{N+n g}$ is not welldefined. However, if we can prove that the theorem holds for any specific representative word in the conjugacy class, then the theorem holds for any representative, because the effect of conjugating $b$ is actually just to change the word $w$.

Proof As discussed in Section 3.4, our strategy will be to carefully piece together a fatgraph using only polygons allowed by Proposition 3.2. In this proof, a partial fatgraph will be a fatgraph with some edges left unattached. That is, a 2-complex whose cells are fatgraph pieces and whose boundary, an oriented simplicial 1-complex, is allowed to contain 1-simplices which are fatgraph edges. 
In the first step, we build a partial fatgraph whose boundary contains the desired word $w$, plus some unglued edges. In the second step, we describe how taking multiple copies of the partial fatgraph allows us to glue the unglued edges to complete the fatgraph in such a way the there is an integer $m$ so that the boundary is multiple loops of the form $\left(w b^{m}\right)^{k}$ for integers $k$. This shows that $w b^{m}$ virtually bounds an immersed surface for some $m$. In the final step, we describe how to vary the power $m$ to arrive at the result.

Let $w=w_{0} \cdots w_{m}$. Without loss of generality, we can assume that $w$ has no cyclic cancellation with $b$. For if it does, we can prepend and append copies of $b$ to $w$ until there is no longer cyclic cancellation ( $b$ has no cancellation with itself), and take $w$ to be this word. These extra copies of $b$ are subsumed into the $N$ in the theorem.

Recall that the standard $\Sigma$ polygon is the polygon $P$ such that $\partial P=O_{\Sigma}$. Because the underlying space of $\Sigma$ is a disk, the boundary word is the product of the finite-order generators. By relabeling, we may assume without loss of generality that $b=c_{0} c_{1} \cdots c_{J-1}$; this implies that the standard $\Sigma$ polygon has (cyclically ordered) boundary $\left[c_{0}, c_{1}, \ldots, c_{J-1}\right]$. This relabeling doesn't affect the proof, but it is simpler to think about. Note that when we start to add infinite-order generators, it will not be true that the arrangement of generators around the standard $\Sigma$ polygon has the same cyclic order as the boundary word.

Step 1 Start with a horizontal polygonal line oriented to the left and labeled by $w$, so the leftmost simplex is labeled by the final letter in $w$. Break $w$ into runs of a single generator. Since $w$ is reduced, any run of $c_{j}$ will have length less than $o_{j}$. Let $W_{1}, \ldots, W_{K}$ denote these runs. For each run $W_{k}$, which will be of the form $W_{k}=c_{j}^{e_{k}}$, build a group polygon which is labeled on top by $W_{k}$, and on the bottom by $o_{j}-e_{k}$ copies of $c_{j}$. This group polygon has $e_{k}-1$ edges on top in between letters in $W_{k}$; these will be glued to monogons so that $c_{j}^{e_{k}}$ appears on the boundary. There are two edges on the left and right between the ends of $W_{k}$ and the first and last new copies of $c_{j}$. These will be glued to other parts of the cyclic fatgraph. Finally, there are $o_{j}-e_{k}-1$ edges on the bottom in between the new copies of $c_{j}$. Onto all of these edges, we attach a copy of the standard $\Sigma$ polygon. These polygons have many edges remaining unglued, and we will return to them later. See Figure 11.

We have one partial fatgraph for every run $W_{k}$, and now we will insert a polygon between successive $W_{k}$ to glue them together. The key is to do this in a way that only uses polygons allowed by Proposition 3.2 and will also allow us to ensure that the boundary other than $w$ is copies of $b$. To glue $W_{k}$ to $W_{k+1}$ (recall $W_{k}$ is to the right of $W_{k+1}$ ), suppose that $W_{k}$ is a run of generator $c_{j}$ and $W_{k+1}$ is a run of generator $c_{l}$. Let $P_{k}$ be the polygon whose boundary $\partial P_{k}$ is the interval in $O_{\Sigma}$ between $c_{l}$ 

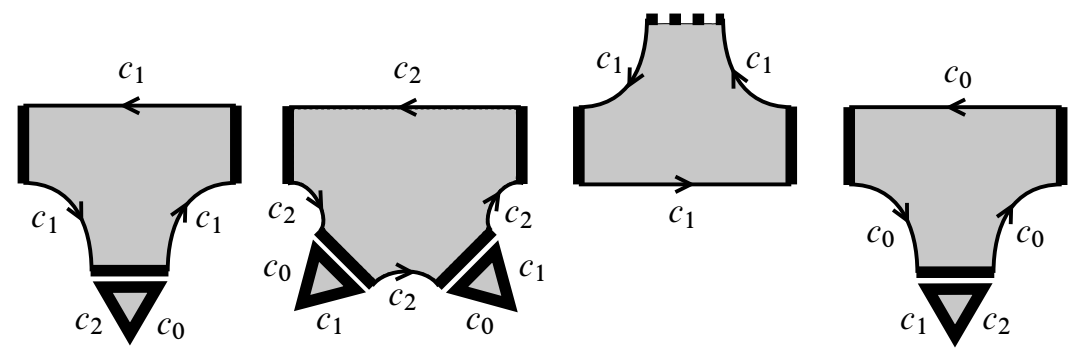

Figure 11: Building a partial cyclic fatgraph for every run $W_{k}$ in $w=$ $c_{0} c_{1}^{2} c_{2} c_{1}$. For this example, we let the generators of $\Gamma$ be $c_{0}, c_{1}, c_{2}$ of orders 3,3 , and 4, respectively, and we take the cyclic order to be $O_{\Sigma}=\left[c_{0}, c_{1}, c_{2}\right]$. These partial fatgraphs are not glued together yet. For clarity, we have labeled the unglued polygon edges with a single letter, so eg pe $\left(c_{j}\right)$ has label $c_{j}$.

and $c_{j}$. That is, $\partial P_{k}$ is a piece of the cyclically ordered set $S$, and $P_{k}$ is simply the standard $\Sigma$ polygon with some edges removed so that $c_{j}$ is followed by $c_{l}$. If $c_{l}$ is immediately followed by $c_{j}$ in $O_{\Sigma}$, then $P_{k}$ will be a bigon.

Now glue every $W_{k}$ to $W_{k+1}$ using $P_{k}$ in the middle. The result is a partial cyclic fatgraph, and observe that along the top, we have the word $w$. On the far left and right ends, there remain two unglued group polygon edges. On the left, build the polygon $P_{K}$ whose boundary is the interval in $O_{\Sigma}$ between $c_{0}$ and $w_{m}$; that is, the first edge of $P_{K}$ is pe $\left(c_{0}\right)$, and the last edge is pe $\left(w_{m}\right)$. For example, if $c_{0}=w_{m}$, then $P_{K}$ will be degenerate (a monogon). If $c_{0}$ follows $w_{m}$ in $O_{\Sigma}$, then $P_{K}$ will be a bigon, and so on. Similarly, build the polygon $P_{0}$ whose boundary is the interval in $O_{\Sigma}$ between $w_{0}$ and $c_{J-1}$. Glue $P_{K}$ and $P_{0}$ on the left and right, respectively. Call the resulting partial fatgraph $Y^{\prime}$. See Figure 12 .

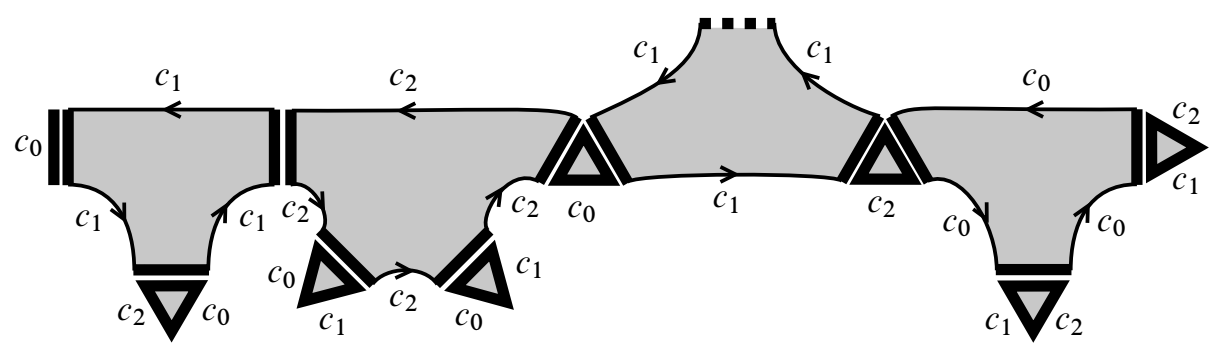

Figure 12: The partial cyclic fatgraph $Y^{\prime}$. Again pe $\left(c_{j}\right)$ is labeled by $c_{j}$.

Step 2 (the covering trick) At this point, we have the fatgraph $Y^{\prime}$, which is composed of group polygons attached around polygons, with some of the polygon edges unglued. Also, all of the polygons have boundary which is an interval in the cyclic order $O_{\Sigma}$, 
so in particular all the polygons are small and have boundary cyclic order compatible with $O_{\Sigma}$. In this step, we show how taking multiple copies of $Y^{\prime}$ makes it possible to glue up all the unglued edges.

Consider what the boundary of $Y^{\prime}$ is. It is an oriented simplicial 1-complex whose simplices are either labeled sides of group polygons or unglued edges from polygons. We claim the following (see Figure 12):

Lemma 4.3 If we read each polygon edge pe $\left(c_{j}\right)$ as the generator $c_{j}$, then $\partial Y^{\prime}=w b^{m}$ for some $m$.

Proof This lemma is really just by construction. Recall that the boundary of the standard $\Sigma$ polygon is the same as the boundary word of $\Sigma$, and every polygon in $Y^{\prime}$ is an interval in $O_{\Sigma}$. Therefore, for any simplex in $\partial Y^{\prime}$ which is not part of $w$ and is labeled pe $\left(c_{j}\right)$ or $c_{j}$, the next simplex must be $\operatorname{pe}\left(c_{j+1}\right)$ or $c_{j+1}$, with indices modulo $J$. If the simplex in $\partial Y^{\prime}$ is part of $w$, then by construction it is a labeled side and is followed by the correct next letter. The special cases of the last letter of $w$ and the last letter of $b$ before $w$ are also correct by construction. Therefore, $\partial Y^{\prime}=w b^{m}$, as desired.

Lemma 4.3 shows that if each unglued polygon edge pe $\left(c_{j}\right)$ is read as $c_{j}$, then the boundary is $w b^{m}$ for some $m$. But of course this isn't enough - we need to produce a complete fatgraph with real boundary. The trick is to take multiple copies of $Y^{\prime}$ and attach group polygons in such a way that the unglued polygon edges are effectively replaced by labeled group polygon sides. We now explain this trick.

Let $L$ be the least common multiple of the $o_{j}$, and take $L$ copies of $Y^{\prime}$. Let $Y^{\prime \prime}$ be these $L$ copies of $Y^{\prime}$, and think of $Y^{\prime \prime}$ as an $L$-sheeted cover of $Y^{\prime}$. The fiber over a single unglued edge pe $\left(c_{j}\right)$ in $Y^{\prime}$ is $L$ copies of pe $\left(c_{j}\right)$. Attach $L / o_{j}$ group polygons to the $L$ unglued edges in $Y^{\prime \prime}$ in the fiber over a single unglued edge in $Y^{\prime}$. Each group polygon can be glued to arbitrary edges within the fiber. Do this for every unglued edge in $Y^{\prime}$. We have attached many group polygons to the partial fatgraph $Y^{\prime \prime}$. Call the result of attaching these group polygons $Y$. We claim:

Lemma 4.4 The result $Y$ is a complete fatgraph whose boundary maps to $w b^{m}$, covering it $L$ times, and contains only small polygons whose boundary is compatible with $O_{\Sigma}$.

Proof Every polygon in $Y$ is a polygon in $Y^{\prime}$, which contained only small polygons with boundary compatible with $O_{\Sigma}$, so the last two conclusions are immediate. Also, $Y$ 


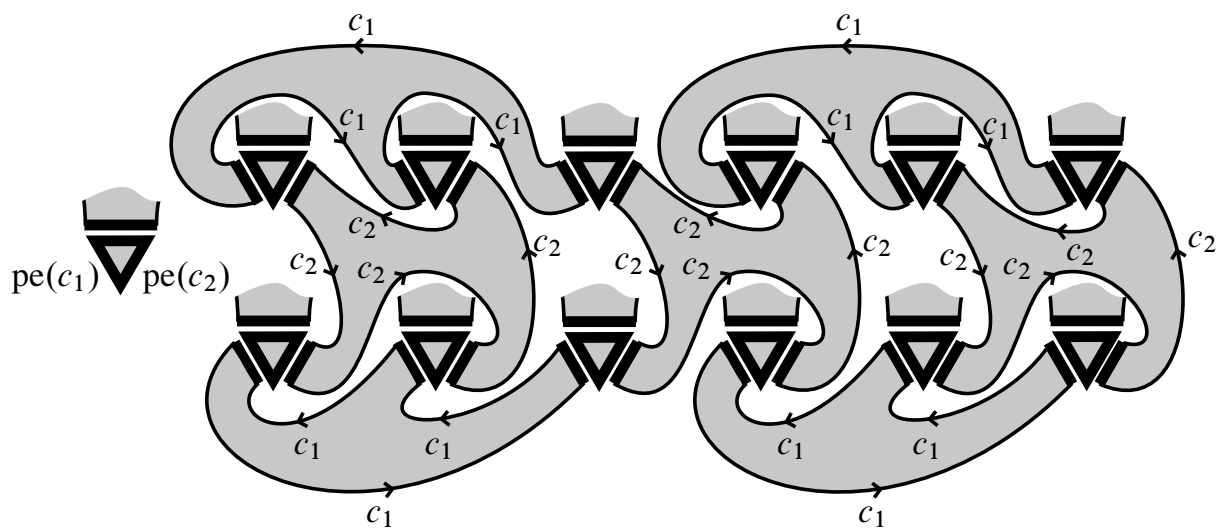

Figure 13: Performing the covering trick on part of the partial fatgraph $Y^{\prime}$ in Figure 12. We take 12 copies of $Y^{\prime}$ and glue group polygons within the fibers. This figure shows what happens to the group polygon on the lower right of Figure 12. The polygon (shown on left) is covered by 12 copies. If we glue in group polygons, note that the boundary has changed from many copies of $\cdots \operatorname{pe}\left(c_{1}\right) \operatorname{pe}\left(c_{2}\right) \cdots$ to many copies of $\cdots c_{1} c_{2} \cdots$.

is created by gluing group polygons to all the unglued edges in $Y^{\prime \prime}$, so it is a complete fatgraph. So the only question is what the boundary of $Y$ is. By Lemma 4.3, the boundary of $Y^{\prime}$ is $w b^{m}$ when we read an unglued edge pe $\left(c_{j}\right)$ as the generator $c_{j}$, so since $Y^{\prime \prime}$ is an $L$-sheeted cover, the same thing is true for each sheet. Now consider $Y$. It is obtained from $Y^{\prime \prime}$ by gluing in group polygons within fibers over each unglued polygon edge in $Y^{\prime \prime}$. So by construction, the boundary of $Y$ is obtained from $\partial Y^{\prime \prime}$ by taking the boundary loops in $Y^{\prime \prime}$ and replacing each unglued polygon edge pe $\left(c_{j}\right)$ with a group polygon side labeled by $c_{j}$ which runs between sheets of $Y^{\prime \prime}$. Thus $\partial Y$ consists of an $L$-degree cover of the loop $w b^{m}$.

Remark 4.5 The $L$-sheeted cover $Y^{\prime \prime}$ of $Y^{\prime}$ has $L$ separate boundary components. When we glue on group polygons to obtain $Y$, each occurrence of an unglued edge $\operatorname{pe}\left(c_{j}\right)$ is replaced by the labeled side $c_{j}$, as we desire. However, this labeled side transits between two different sheets of $Y^{\prime \prime}$. Therefore, the final boundary of $Y$ will look locally as if we simply replaced $\operatorname{pe}\left(c_{j}\right)$ with $c_{j}$, but some of the boundary loops may have been joined together. The total degree remains $L$.

For example, to perform this "covering trick" on the partial fatgraph $Y^{\prime}$ in Figure 12, we would compute the least common multiple of the $o_{j} 3,3$, and 4, which is 12 . Take 12 copies of the partial fatgraph to get $Y^{\prime \prime}$. Now for any unglued polygon edge in $Y^{\prime}$, glue group polygons to the unglued edges in $Y^{\prime \prime}$ in the fiber. See Figure 13. 
Because $Y$ contains only polygons which are compatible with the cyclic order $O_{\Sigma}$, we can apply Proposition 3.2 to show that $f_{Y}$ is homotopic to an immersion with geodesic boundary, so $w b^{m}$ virtually bounds an immersed surface. This completes Step 2 .

Step 3 We need more than the fact that $w b^{m}$ virtually bounds an immersed surface: Theorem 4.1 is a stability result, and we need to show that there is $N$ such that for all $n>0$, we have that $w b^{N+n g}$ virtually bounds an immersed surface, where $g=\operatorname{gcd}\left(o_{0}-1, \ldots, o_{J-1}-1\right)$. In Step 2, we showed that for some $m, w b^{m}$ virtually bounds an immersed surface. In this step, we show that we can actually achieve any desired $m$, as long as it is large enough and $g \mid m$.

Consider again the partial fatgraph $Y^{\prime}$ from Step 2. By Lemma 4.3, if we read the unglued polygon edge pe $\left(c_{j}\right)$ as $c_{j}$, then the boundary of $Y^{\prime}$ is $w b^{m}$ for some $m$. For the current step, we need there to be some unglued polygon edge. This is almost certainly the case, but if not, append a copy of $b$ onto $w$, which forces some unglued edges. So without loss of generality, we assume there is an unglued polygon edge, and also without loss of generality, we assume it is pe $\left(c_{0}\right)$. We will now re-use notation and define a new $Y^{\prime \prime}$ for this step. Let $Y^{\prime \prime}$ be the partial fatgraph obtained from $Y^{\prime}$ by attaching a $c_{0}$-group polygon onto the unglued polygon edge pe $\left(c_{0}\right)$ and attaching standard $\Sigma$ polygons onto all unglued edges of this new group polygon.

Note that we have added $o_{0}-1$ new polygons, and, reading unglued edges pe $\left(c_{j}\right)$ as $c_{j}$, the boundary of $Y^{\prime \prime}$ is $w b^{m+\left(o_{0}-1\right)}$. Also note that the newly attached standard $\Sigma$ polygons have every edge unglued except $c_{0}$. So for any $j$ except $j=0$, we can repeat this procedure to obtain a fatgraph whose boundary is $w b^{m+\left(o_{0}-1\right)+\left(o_{j}-1\right)}$ (when unglued edges are read as generators). See Figure 14.

Therefore, by repeating this procedure, we can obtain a partial fatgraph whose boundary (with pe $\left(c_{j}\right)$ read as $\left.c_{j}\right)$ is $w b^{M}$, where $M$ is any integer of the form $m+\sum_{k=1}^{K}\left(o_{i_{k}}-\right.$ 1), where the successive $i_{k}$ are distinct. By Lemma 4.6, there is some $N$ such that for all $n \geq 0$, every integer $N+n g$ is of this form. Now take this partial fatgraph with boundary $w b^{M}$ and perform Step 2 (the covering trick) to get a real, complete fatgraph satisfying Proposition 3.2. This shows that $w b^{M}$ virtually bounds an immersed surface, and completes the proof.

The following lemma is required by the proof of Theorem 4.1, but it is independently interesting.

Lemma 4.6 Given integers $\left\{x_{i}\right\}_{i=1}^{k}$ with $k \geq 3$ and with $g=\operatorname{gcd}_{i} x_{i}$, there is some $N \in \mathbb{N}$ so that for all $n \in \mathbb{N}$, there is an integer sequence $\left\{i_{j}\right\}_{j=1}^{J}$ such that $i_{j} \neq i_{j+1}$ for all $j$ and $\sum_{j=1}^{J} x_{i_{j}}=N+n g$. 


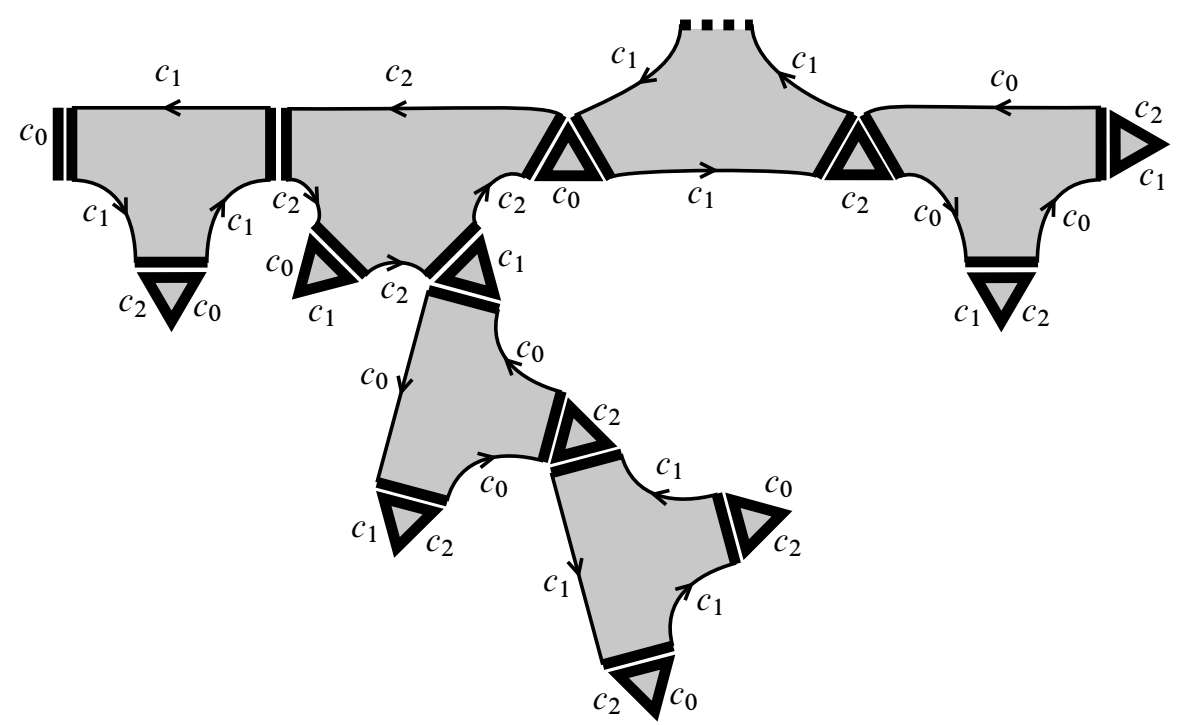

Figure 14: Adding group polygons to add copies of $b$ to the boundary. Notice that upon adding the first group polygon, the newly introduced standard $\Sigma$ polygons have every possible unglued edge except $e\left(c_{0}, c_{0}\right)$, to which we attached the first group polygon.

Proof We call the sequence $1,2, \ldots, k$ a run. The idea in constructing the sequence $i_{j}$ is to start with a repeating sequence of runs $1,2, \ldots, k, 1,2, \ldots, k, \ldots$ and take away a single number from every other run. The resulting list will have distinct adjacent pairs. We can write $g=\sum_{i=1}^{k} a_{i} x_{i}$. Let $s=\sum_{i=1}^{k} x_{i}$, and let $M=\max _{i} a_{i}$ and $m=\min _{i} a_{i}$. Let $N=2 k s(s / g)(M-m)$. Now, given $C>N$ such that $g \mid C$, write $C=c s+r$, where $r<s$. Notice that

- $c \geq 2 k(s / g)(M-m)$, and

- $g \mid r$ because $g \mid s$, so

- $r=d g$, where $d<s / g$.

Therefore, we can rewrite

$$
\begin{aligned}
C=c s+d g=c s+d \sum_{i=1}^{k} a_{i} x_{i} & =c s+d M \sum_{i=1}^{k} x_{i}-d \sum_{i=1}^{k}\left(M-a_{i}\right) x_{i} \\
& =(c+d M) s-\sum_{i=1}^{k} d\left(M-a_{i}\right) x_{i},
\end{aligned}
$$

where every term $M-a_{i}$ is non-negative. Start with a sequence of $(c+d M)$ runs. If we could remove $d\left(M-a_{i}\right)$ copies of $i$ from the sequence, for each $i$, then 
$\sum_{j} x_{i_{j}}$ for the resulting sequence $\left\{i_{j}\right\}_{j}$ would have the correct value $C$. But we must be certain that when these copies of $i$ are removed, the adjacent elements in the sequence remain distinct. To accomplish this, we can remove a single $i$ from every other run. We need to know that we have enough runs available, ie we need at least $2 d\left(M-a_{i}\right) \leq 2 d(M-m) \leq 2(s / g)(M-m)$ runs for every $i$. But from our bound on $c$, we have at least $c \geq 2 k(s / g)(M-m)$ runs, so we can remove the indices as desired, and the proof is complete.

Remark 4.7 Theorem 4.1 generalizes [5, Theorem 3.1]. The situation of interest in [5] is $(2, p, \infty)$ orbifolds, which have only two orbifold points, and as stated, Theorem 4.1 requires 3 orbifold points. However, in the special case of two orbifold points with one point of order 2, Lemma 4.6 can be avoided, and Theorem 4.1 still goes through. The proof of Theorem 4.1 is essentially a combinatorialization of the argument in [5].

\subsection{Orbifolds with genus}

We now prove an analog of Theorem 4.1 in the case that the orbifold has genus at least 1 . In this case, we can avoid any number-theoretic issues.

Theorem 4.8 Let $\Sigma$ be a hyperbolic orbifold with one boundary component and with genus at least 1 . Let $\Gamma=\pi_{1}(\Sigma)$ with $b=\partial \Sigma \in \Gamma$, and let $w \in \Gamma$ be hyperbolic so that some power of $w$ is homologically trivial. Then there exists $N \in \mathbb{N}$ so that for all $n \geq 0$, the loop representing $w b^{N+n}$ virtually bounds an immersed surface with geodesic boundary.

Proof As with the disk orbifold proof, in Step 1, we construct a partial fatgraph with some unglued polygon edges. The proof becomes different in Step 2: We cannot use a covering trick to fill in unglued polygon edges for infinite-order generators. Therefore, we exhibit small partial fatgraph modules which can be inserted to fill in these edges. Then we use the covering trick to fill in all the finite-order edges.

After relabeling, we can assume that the cyclic order on the infinite order generators is such that the boundary has the standard form $\left[z_{0}, z_{1}\right] \cdots\left[z_{I-2}, z_{I-1}\right]$ (if there are finite-order generators, they are inserted within this cyclic boundary word). Therefore, the cyclic order is $\left[z_{0}, z_{1}^{-1}, z_{0}^{-1}, z_{1}, \cdots, z_{I-2}, z_{I-1}^{-1}, z_{I-2}^{-1}, z_{I-1}\right]$ (with finite-order generators inserted at appropriate positions). Note that the cyclic order isn't the same as the boundary word. It will be useful to be able to refer to the letters in $w$ and the boundary word $b$, but their lengths will not matter. Therefore, we use $w_{0}$ and $w_{-1}$ to refer to the first and last letters in $w$, and similarly for $b$. 
Step 1 Perform Step 1 as in the proof of Theorem 4.1 to get a partial cyclic fatgraph $Y^{\prime}$ which has boundary $w$ along the top and many unglued polygon edges. There are two situations not covered by those instructions, as follows. First, each infinite order generator $z_{i}$ is in a run $W_{k}$ by itself, and the fatgraph piece we use for this run is simply a rectangle. Second, when building the polygon to be glued onto the far left, we use the polygon which is the interval in $O_{\Sigma}$ between $b_{0}$ and $w_{-1}$, and the polygon for the far right is the interval between $w_{0}$ and $b_{-1}$. In the proof of Theorem 4.1, we used $c_{0}$ and $c_{J-1}$ in place of $b_{0}$ and $b_{-1}$ because in the disk orbifold case we know that the boundary word is exactly $c_{0} \cdots c_{J-1}$. See Figure 15 for an example of $Y^{\prime}$.

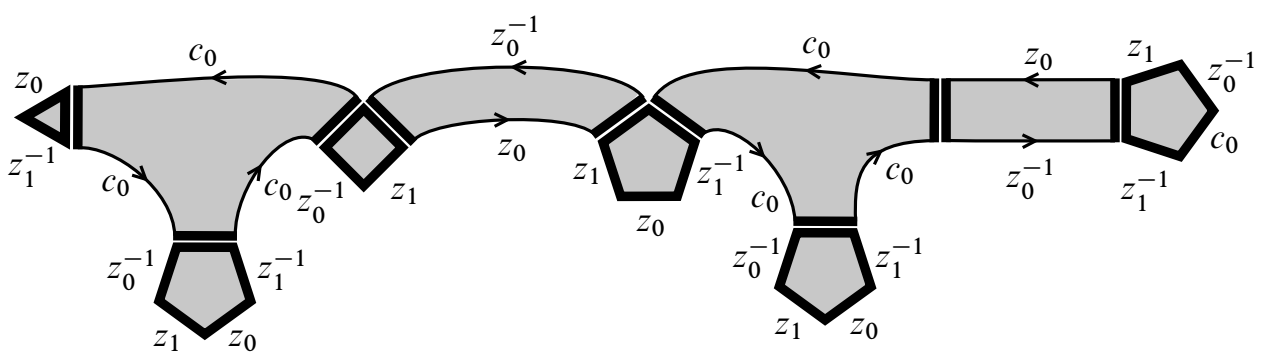

Figure 15: The partial fatgraph $Y^{\prime}$ for the word $w=z_{0} c_{0} z_{0}^{-1} c_{0}$ in an orbifold of genus 1 with one orbifold point of order 3 , with cyclic order $\left[z_{0}, z_{1}^{-1}, c_{0}, z_{0}^{-1}, z_{1}\right]$ and thus boundary $b=z_{0} z_{1} c_{0} z_{0}^{-1} z_{1}^{-1}$. For simplicity, we denote the edges $\operatorname{pe}\left(z_{i}\right)$ and $\operatorname{pe}\left(c_{j}\right)$ by $z_{i}$ and $c_{j}$, respectively. This picture omits the replacement of $w$ by $w b^{2}$, as described at the end of Step 1, because it is not necessary to do that to have enough unglued polygon edges in this example.

For reasons which become apparent in Step 3, we need for there to be sufficiently many unglued polygon edges. Therefore, we assume that we have appended a copy of $b^{2}$ onto $w$. Since the polygon inserted between letters in $b$ is a complete standard $\Sigma$ polygon, and $b$ has length at least 4 , we ensure that there are at least four polygon edges pe $\left(z_{i}\right)$ and four polygon edges $\operatorname{pe}\left(z_{i}^{-1}\right)$ for each $i$. For simplicity, we will not show this in our example pictures.

Step 2 In this step, we describe how to fill in the unglued polygon edges associated with the infinite-order generators. We do this by building small partial fatgraph "modules" which can be glued in to complete $Y^{\prime}$.

It will be convenient to be able to refer to subwords of the boundary word $b$. Denote by $b_{i,+}$ and $b_{i,-}$ the subword of the (cyclic) word $b$ between (not including) $z_{i}$ and $z_{i}^{-1}$, and $z_{i}^{-1}$ and $z_{i}$, respectively. For example, if $b=z_{0} z_{1} c_{0} z_{0}^{-1} z_{1}^{-1}$, then $b_{0,+}=z_{1} c_{0}$ and $b_{0,-}=z_{1}^{-1}$. 
Now let $P$ be the standard $\Sigma$ polygon, whose boundary is $O_{\Sigma}$. For each infinite-order generator $z_{i}$, attach the rectangle $r\left(z_{i}\right)$ to both polygon edges $\operatorname{pe}\left(z_{i}\right)$ and $\mathrm{pe}\left(z_{i}^{-1}\right)$. Call this partial fatgraph $A$. Note that if we read unglued finite-order polygon edges $\operatorname{pe}\left(c_{j}\right)$ as $c_{j}$, then $\partial A=b$; ie the boundary of $A$ is the boundary word of $\Sigma$.

For each $i$, define a partial fatgraph $A_{i}$ as follows. Detach one edge of the rectangle $r\left(z_{i}\right)$ from the polygon $P$, and glue a duplicate copy of $r\left(z_{i}\right)$ to the polygon edge which is now unglued. That is, instead of there being a single rectangle with both edges glued to $P$, there are now two rectangles, each of which is glued to one of the two edges $\operatorname{pe}\left(z_{i}\right)$ and $\operatorname{pe}\left(z_{i}^{-1}\right)$ in $P$. See Figure 16.

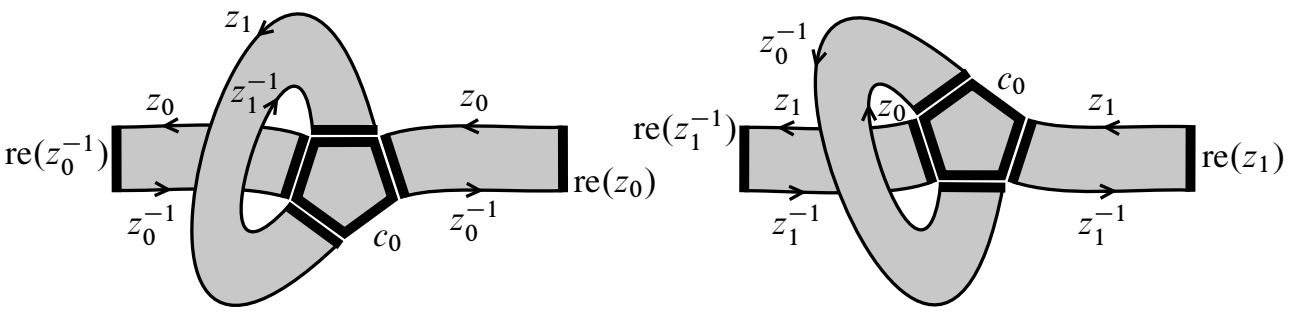

Figure 16: The partial fatgraph modules $A_{0}$ and $A_{1}$ in the example from Figure 15

Lemma 4.9 The partial fatgraph $A_{i}$ has two boundary components. When finite-order polygon edges pe $\left(c_{j}\right)$ are read as $c_{j}$, the 1 -simplices in the boundary are labeled $\operatorname{pe}\left(z_{i}\right) z_{i} b_{i,+} z_{i}^{-1}$ and $\operatorname{pe}\left(z_{i}^{-1}\right) z_{i}^{-1} b_{i,-} z_{i}$. Consequently, there are no infinite-order unglued polygon edges, and there are exactly the two unglued rectangle edges re $\left(z_{i}\right)$ and $\operatorname{re}\left(z_{i}^{-1}\right)$.

Proof This is just by construction; Figure 16 illustrates it. Suppose we build a fatgraph $B$ by attaching the bigon $\left[\mathrm{pe}\left(z_{i}\right), \mathrm{pe}\left(z_{i}^{-1}\right)\right]$ to the two unglued rectangle edges in $A_{i}$. Then we have effectively taken $A$ and replaced the rectangle $r\left(z_{i}\right)$ with two rectangles glued end-to-end. Therefore, the boundary of $B$ is $b$ with $z_{i}$ and $z_{i}^{-1}$ duplicated. So the boundary of $B$ is $z_{i}^{2} b_{i,+} z_{i}^{-2} b_{i,-}$. Now remove the bigon to get $A_{i}$ back from $B$, which cuts the boundary in two at the $z_{i}^{2}$ and $z_{i}^{-2}$ and inserts the rectangle edges as claimed.

The partial fatgraphs $A_{i}$ are the small modules which we will glue onto $Y^{\prime}$ to fill in the unglued polygon edges. Each $A_{i}$ has the two rectangle edges $\operatorname{re}\left(z_{i}\right)$ and $\operatorname{re}\left(z_{i}^{-1}\right)$. So we must verify that $Y^{\prime}$ contains the same number of $\operatorname{pe}\left(z_{i}\right)$ as $\operatorname{pe}\left(z_{i}^{-1}\right)$ for each $i$.

Lemma 4.10 For every $i, Y^{\prime}$ contains the same number of unglued polygon edges $\operatorname{pe}\left(z_{i}\right)$ and $\mathrm{pe}\left(z_{i}^{-1}\right)$. 
Proof The purpose of the this lemma is to verify that we can attach the partial fatgraphs $A_{i}$ to $Y^{\prime}$ to fill in all the unglued polygon edges. So it is interesting that to prove this lemma, we will attach different partial fatgraphs to $Y^{\prime}$ and then make some observations about the result. It is also possible to prove the lemma with some technical combinatorial counting, but this method is more intuitive.

Note that the infinite-order generators naturally come in pairs, one for each genus. For each $i$, let $i^{\prime}$ denote the index with which $i$ is paired. So $i^{\prime}=i+1$ if $i \equiv 0 \bmod 2$, and $i^{\prime}=i-1$ if $i \equiv 1 \bmod 2$.

Consider the partial fatgraph $A_{i}$. It has two unglued rectangle edges $\operatorname{re}\left(z_{i}\right)$ and $\operatorname{re}\left(z_{i}^{-1}\right)$. Because the fundamental group of $A_{i}$ embeds in $\Gamma$, we will refer to elements of $\pi_{1}\left(A_{i}\right)$ by their images in $\Gamma$. Because we have assumed the standard form for the generators, a loop freely homotopic to $z_{i^{\prime}}$ in $A_{i}$ is separating, and the two boundary components of $A_{i}$ are in different connected components of the complement of the loop $z_{i^{\prime}}$. See Figure 17.

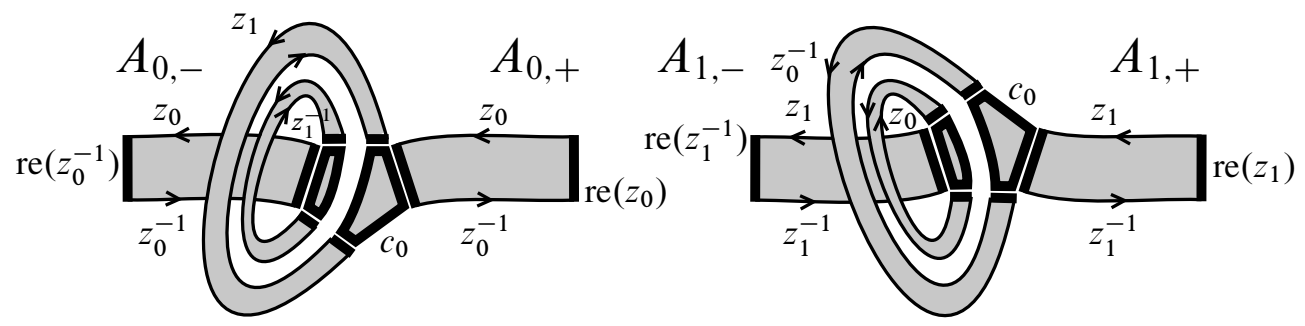

Figure 17: The fatgraph modules $A_{0}$ and $A_{1}$ as in Figure 16, get cut into the modules $A_{0, \pm}$ and $A_{1, \pm}$ in the proof of Lemma 4.10

Thus the loop $z_{i^{\prime}}$ cuts $A_{i}$ into two surfaces, which we will refer to as $A_{i,+}$ and $A_{i,-}$. The surface $A_{i,+}$ has two boundary components, the loops $\operatorname{re}\left(z_{i}\right) z_{i} b_{i,+} z_{i}^{-1}$ and $z_{i^{\prime}}^{ \pm 1}$, and $A_{i,-}$ has the boundaries $\operatorname{re}\left(z_{i}^{-1}\right) z_{i}^{-1} b_{i,-} z_{i}$ and $z_{i^{\prime}}^{\mp 1}$. We use the symbols $z_{i^{\prime}}^{ \pm 1}$ and $z_{i^{\prime}}^{\mp 1}$ to refer to the positive and negative powers of $z_{i^{\prime}}$ because which power goes with $A_{i,+}$ and $A_{i,-}$ depends on the parity of $i$. This doesn't matter; the key fact is that $A_{i,+}$ and $A_{i,-}$ each have a $z_{i}$ boundary loop, and the loops on $A_{i,+}$ and $A_{i,-}$ have opposite signs.

So for each $i$, we have the partial fatgraphs $A_{i,+}$ and $A_{i,-}$, which have the single unglued rectangle edge $\operatorname{re}\left(z_{i}\right)$ and $\operatorname{re}\left(z_{i}^{-1}\right)$, respectively. Build a new fatgraph $X$ by attaching a copy of $A_{i, \pm}$ to every unglued infinite-order polygon edge $\operatorname{pe}\left(z_{i}^{ \pm 1}\right)$ in $Y^{\prime}$. Each $A_{i, \pm}$ has only a single unglued rectangle edge, so there is no obstruction to attaching them to every unglued edge. Because the $A_{i, \pm}$ have no unglued infiniteorder polygon edges, there are no infinite-order unglued polygon edges in $X$, so $X$ 
contains only labeled sides of rectangles and unglued finite-order polygon edges. And by the construction of $Y^{\prime}$ (because every polygon in $Y^{\prime}$ is an interval in $O_{\Sigma}$ ), if we read each finite-order edge $\operatorname{pe}\left(c_{j}\right)$ as $c_{j}$, the boundary of $X$ is of the form $w b^{m}+$ $\sum_{i=0}^{I-1} M_{i} z_{i}+m_{i} z_{i}^{-1}$ for integers $m, M_{i}$, and $m_{i}$. The $z_{i}^{ \pm 1}$ boundary components arise from the small boundary loops in the $A_{i, \pm}$. Because the only unglued edges in $X$ are finite-order edges, the boundary of $X$ has a finite power which is homologically trivial. And since $w$ and $b$ both have finite powers which are homologically trivial, the sum $\sum_{i=0}^{I-1} M_{i} z_{i}+m_{i} z_{i}^{-1}$ must be homologically trivial, so it must be that $m_{i}=M_{i}$ for each $i$. But these integers count the number of copies of $A_{i,+}$ and $A_{i,-}$ which we attached to $Y^{\prime}$, so we conclude that the number of unglued edges $\operatorname{pe}\left(z_{i}\right)$ is equal to the number of unglued edges $\operatorname{pe}\left(z_{i}^{-1}\right)$, as desired.

By Lemma 4.10, the partial fatgraph $Y^{\prime}$ contains the same number of edges pe $\left(z_{i}\right)$ as $\operatorname{pe}\left(z_{i}^{-1}\right)$ for each $i$. Call this number $m_{i}$. Therefore, it is possible to attach $m_{i}$ copies of $A_{i}$ to $Y^{\prime}$ for each $i$. Call the resulting fatgraph $Y^{\prime \prime}$. By construction, $Y^{\prime \prime}$ has a single boundary component, which, if $\operatorname{pe}\left(c_{j}\right)$ is read as $c_{j}$, is $w b^{m}$ for some $m$. By applying Step 2 of Theorem 4.1, the covering trick, to $Y^{\prime \prime}$ we can produce a complete fatgraph $Y$ whose boundary covers $w b^{m}$. By construction $Y$ contains only polygons whose boundaries are intervals of $O_{\Sigma}$, so they are small and compatible with $O_{\Sigma}$. Therefore, the existence of $Y$ shows that $w b^{m}$ virtually bounds an immersed surface in $\Sigma$. Figure 18 shows the result $Y^{\prime \prime}$ of attaching all the $A_{i}$ to the partial fatgraph $Y^{\prime}$ shown in Figure 15. This completes Step 2.

Step 3 As with the proof of Theorem 4.1, we have shown that $w b^{m}$ virtually bounds an immersed surface for some $m$, but we need to show the stability result that there is an $N$ such that for all $n \geq 0, w b^{N+n}$ virtually bounds an immersed surface.

To prove this, we will construct some new partial fatgraph "modules" which can replace some of the $A_{i}$ and have the effect of increasing the power of $b$ in the boundary. As it turns out, it is quite simple to increase the power of $b$ by two, which would prove the theorem for even $n$. It is more complicated to increase the power by one; this requires taking a cover.

First, we show how to increase the power of $b$ by (a multiple of) two. This requires exhibiting a new kind of partial fatgraph, which we will denote by $A_{i, k}$. Recall that $i^{\prime}$ is the generator paired with $i$, so that $\left[z_{i}, z_{i^{\prime}}\right]^{ \pm 1}$ appears in $b$, possibly with finite-order generators inserted. Given $k$ even, build $A_{i, k}$ as follows (See Figure 19): take $k+1$ copies of the standard $\Sigma$ polygon, indexed by $P_{\ell}$ for $\ell=0 \ldots k$. For every infiniteorder generator $z_{t}$ with $t \neq i$ and $t \neq i^{\prime}$, add $k+1$ copies of the rectangle $r\left(z_{t}\right)$, each one connected at both edges to a single polygon $P_{\ell}$. Next, add $k+2$ copies of $r\left(z_{i}\right)$. One copy has rectangle edge $\operatorname{re}\left(z_{i}\right)$ connected to $P_{0}$; one copy has rectangle 


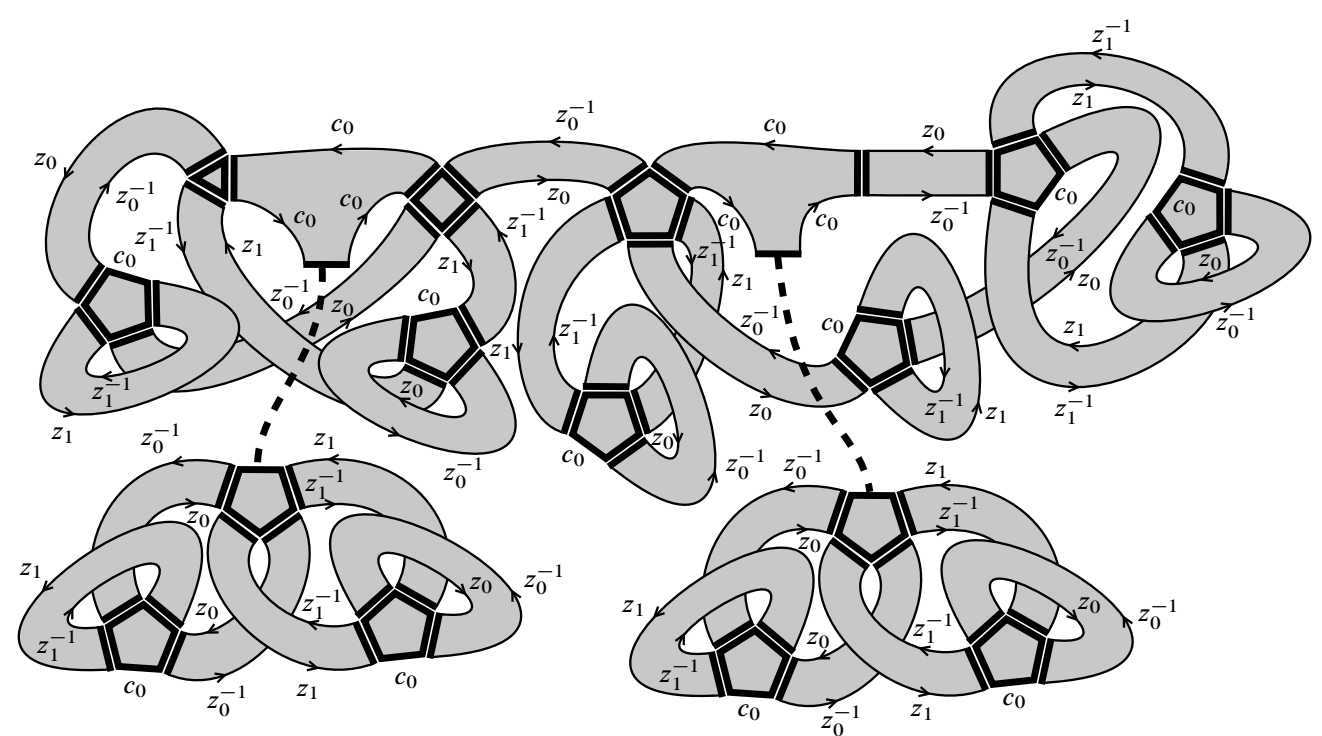

Figure 18: The result of attaching the $A_{i}$ to the partial fatgraph in Figure 15. For clarity, two regions of the fatgraph are drawn separately, and dotted lines indicate where they should be placed. Patience reveals that the boundary is, in fact, $w b^{14}$.

edge re $\left(z_{i}^{-1}\right)$ connected to $P_{k}$; and the remaining copies connect $P_{\ell}$ to $P_{\ell+1}$. Finally, add $k+1$ copies of $r\left(z_{i^{\prime}}\right)$, as follows: for each $\ell$ divisible by 2, add two copies of $r\left(z_{i^{\prime}}\right)$ which connect $P_{\ell}$ to $P_{\ell+1}$. The boundary of $A_{i, k}$ has two components by construction, with the labels $\operatorname{re}\left(z_{i}^{-1}\right) z_{i}^{-1} b^{k} b_{i,-} z_{i}$ and $\operatorname{re}\left(z_{i}\right) z_{i} b_{i,+} z_{i}^{-1}$. Note the power $b^{k}$ in the first boundary component is the cyclic word $b^{k}$; it may be cyclically rotated from the original choice of a cyclic representative that we called $b$. This is correct, since if we insert a copy of $b$ in the middle of a power of $b$, we must cyclically shift the inserted copy, depending on the location it is inserted, so that it aligns correctly. See Figure 19.

Note that the boundary of $A_{i, k}$ is exactly that of $A_{i}$, except one of the boundaries has $k$ copies of $b$ inserted. Thus, if we replace one of the copies of $A_{i}$ for some $i$ with $A_{i, k}$ in Step 2, the resulting fatgraph $Y$ has boundary $w b^{m+k}$. The fact that we can insert a copy of $A_{i, k}$ requires that we have at least two unglued polygon edges. Recall we ensured this in Step 1. This shows that $w b^{m+k}$ virtually bounds an immersed surface for any even $k$.

Finally, we show how to build a fatgraph with boundary $w b^{m+k+1}$ for any even $k$. Consider again $Y^{\prime}$, and recall that $Y^{\prime}$ has at least four unglued polygon edges pe $\left(z_{0}\right)$ and $\operatorname{pe}\left(z_{0}^{-1}\right)$ (it has these unglued edges for every $i$; we choose 0 arbitrarily). Glue copies of $A_{i}$ to all unglued polygon edges for every index except 0 , and glue a copy 


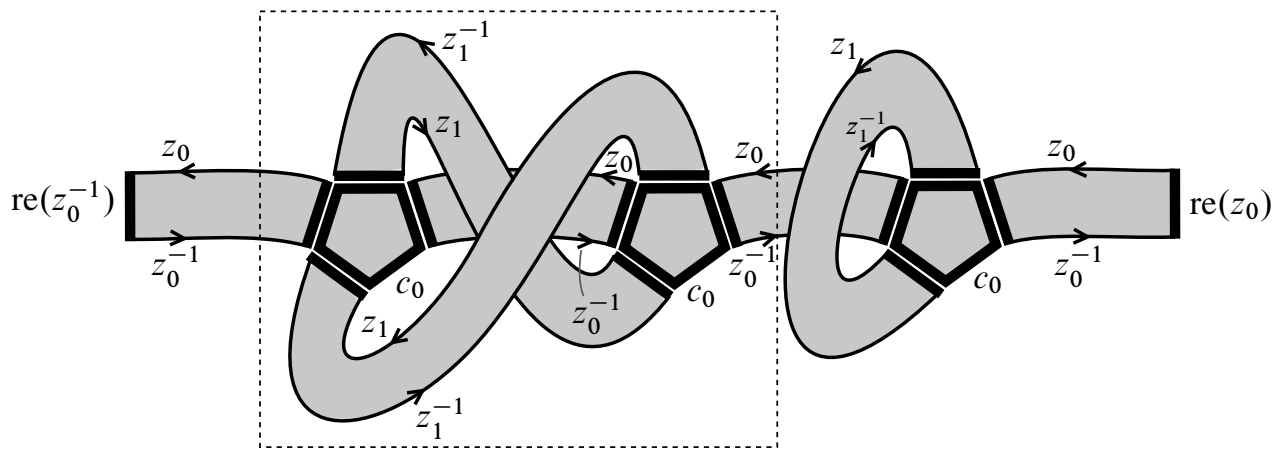

Figure 19: The partial fatgraph $A_{0,2}$. The boxed area is duplicated as desired to produce $A_{0, k}$. Note that replacing one of the $A_{0}$ with $A_{0, k}$ increases the power of $b$ in the boundary by $k$.

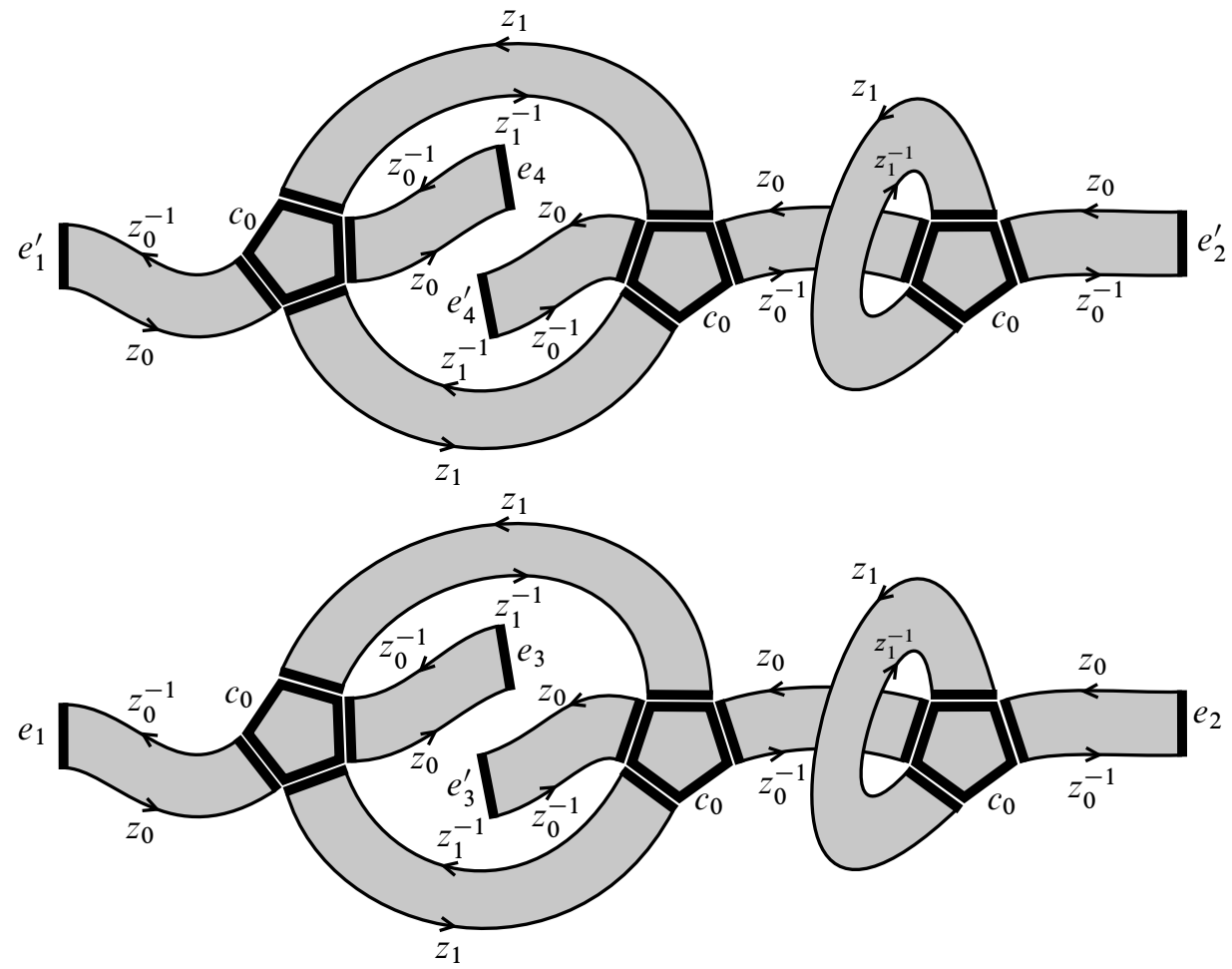

Figure 20: Two copies of the partial fatgraph module $B$, with rectangle edges labeled as they should be attached to $X^{\prime}$ in the proof of Theorem 4.8

of $A_{0}$ to one of the four pairs of unglued edges for index 0 , leaving three pairs. Next, attach a copy of $A_{0, k}$ to one of the pairs, leaving two pairs. Call the resulting fatgraph $X$. Note $X$ has exactly four unglued polygon edges: two pe $\left(z_{0}\right)$, which we denote 
by $e_{1}$ and $e_{2}$ and two pe $\left(z_{0}^{-1}\right)$, which we denote by $e_{3}$ and $e_{4}$. Let $X^{\prime}$ be the partial fatgraph which is two copies of $X$, and think of $X^{\prime}$ as a double cover of $X$. Each $e_{s}$ has two edges covering it, which we denote by $e_{s}$ and $e_{S}^{\prime}$.

We are going to attach two copies of a fatgraph module $B$ to the unglued edges in $X^{\prime}$. The module $B$ is similar to $A_{0,2}$, and is created from $A_{0,2}$ by removing the $r\left(z_{0}\right)$ rectangle between polygons 0 and 1 in the construction of $A_{0,2}$ and replacing it with two rectangles, one glued to polygon 0 and one glued to polygon 1 . This leaves four unglued rectangle edges. It is far easier to understand by consulting Figure 20. Though this picture is for a specific example, $B$ in any other case is formed by just adding finite-order edges and genus loop pairs at locations on the standard polygons; it doesn't actually change the form of the module.

Take two copies of $B$, as shown in Figure 20, and attach the edges to $X^{\prime}$ as labeled to produce a fatgraph $X^{\prime \prime}$. Note $X^{\prime \prime}$ has no unglued infinite-order polygon edges, and reading $\operatorname{pe}\left(c_{j}\right)$ as $c_{j}$, we find that the boundary of $X^{\prime \prime}$ is two copies of $w b^{m+k+1}$.

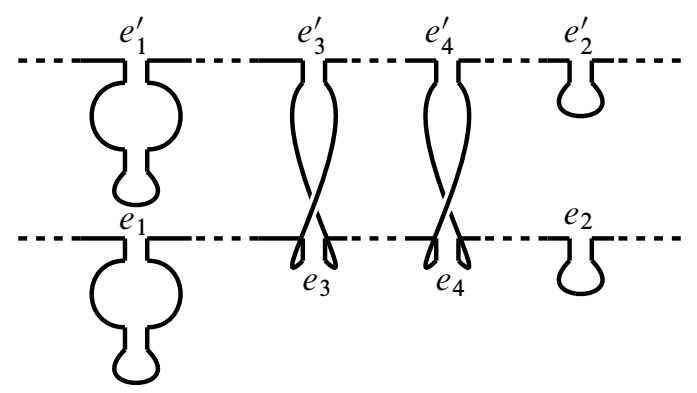

Figure 21: A schematic showing the boundary of $X^{\prime \prime}$ after attaching two copies of $B$ to $X^{\prime}$. The two levels indicate the two sheets of $X^{\prime}$ as a cover of $X$. This picture shows why this particular way of attaching the edges adds one copy of $b$ in the same place on both boundaries of the cover.

Figure 21 shows a schematic of how the boundary behaves after attaching the two copies of $B$. The exact arrangement of edges used in attaching $B$ to $X^{\prime}$ is important: we need a fatgraph whose boundary is two copies of $w b^{m+k+1}$. Were we to attach differently, we would have a fatgraph whose boundary contained two copies of $w$ and many copies of $b$, but the powers of $b$ in between the $w$ might not be the same. Attaching as instructed places the extra copy of $b$ in the same place on both sheets of the cover $X^{\prime}$.

Now performing the covering trick on $X^{\prime \prime}$ produces a fatgraph $Y$ whose boundary covers $w b^{m+k+1}$, and by construction $Y$ satisfies Proposition 3.2. We have now shown that $w b^{m+k}$ and $w b^{m+k+1}$ virtually bound immersed surfaces for every even $k$, so this completes the proof. 
Remark 4.11 Theorem 4.8 applies in the case of a hyperbolic surface with a single boundary (and no orbifold points), so it resolves [2, Conjecture 3.16].

Remark 4.12 When a loop $\gamma$ virtually bounds an immersed surface, it means there is an immersed fatgraph with geodesic boundary whose boundary covers $\gamma$ with some degree, which we call the covering degree of $\gamma$. The proofs of Theorems 4.1 and 4.8 show that the covering degree of $w b^{n}$ depends on the orders of the finite-order generators and is independent of $w$ and $n$. In particular, if there are no orbifold points, then the covering degree is either 1 or 2 depending on the parity of $n$.

\section{References}

[1] D Calegari, Foliations and the geometry of 3-manifolds, Oxford University Press (2007) MR2327361

[2] D Calegari, Faces of the scl norm ball, Geom. Topol. 13 (2009) 1313-1336 MR2496047

[3] D Calegari, scl, MSJ Memoirs 20, Math. Soc. Japan, Tokyo (2009) MR2527432

[4] D Calegari, Stable commutator length is rational in free groups, J. Amer. Math. Soc. 22 (2009) 941-961 MR2525776

[5] D Calegari, J Louwsma, Immersed surfaces in the modular orbifold, Proc. Amer. Math. Soc. 139 (2011) 2295-2308 MR2784794

[6] M Culler, Using surfaces to solve equations in free groups, Topology 20 (1981) 133145 MR605653

[7] W P Thurston, The geometry and topology of three-manifolds, lecture notes, Princeton University (1979) Available at http://msri.org/publications/books/gt3m

[8] A Walker, Stable commutator length in free products of cyclic groups, Exp. Math. 22 (2013) 282-298 MR3171093

Department of Mathematics, University of Chicago,

5734 S University Avenue, Chicago, IL 60637, USA

akwalker@math.uchicago .edu

http://math.uchicago.edu/ akwalker

Received: 7 January $2014 \quad$ Revised: 20 November 2014 